\title{
CONVERGENCE TO STATIONARY STATES \\ IN THE MAXWELL-BLOCH SYSTEM FROM NONLINEAR OPTICS
}

BY

\author{
FRANK JOCHMANN
}

Institut für angewandte Mathematik, Humboldt Universität, Berlin

1. Introduction. The subject of this paper is the Maxwell-Bloch equations from nonlinear optics:

$$
\partial_{t} \mathbf{E}=\operatorname{curl} \mathbf{H}-\partial_{t} \widetilde{\mathbf{P}}-\mathbf{j}-\sigma \mathbf{E}, \quad \partial_{t} \mathbf{H}=-\operatorname{curl} \mathbf{E},
$$

on $\mathbb{R}^{+} \times \Omega$ coupled with the equations

$$
\partial_{t}^{2} \mathbf{P}+\beta \partial_{t} \mathbf{P}+\alpha \mathbf{P}=N \mathbf{E}
$$

on $\mathbb{R}^{+} \times G$ and

$$
\partial_{t} N+\lambda\left(N-N_{e}\right)=-\gamma \mathbf{E} \partial_{t} \mathbf{P}
$$

also on $\mathbb{R}^{+} \times G$. The initial boundary conditions

$$
\begin{gathered}
\vec{n} \wedge \mathbf{E}=0 \text { on }(0, \infty) \times \Gamma_{1} \quad \text { and } \quad \vec{n} \wedge \mathbf{H}=0 \text { on }(0, \infty) \times \Gamma_{2}, \\
\mathbf{E}(0, x)=\mathbf{E}_{0}(x), \quad \mathbf{H}(0, x)=\mathbf{H}_{0}(x), \\
N(0, x)=N_{0}(x), \quad \mathbf{P}(0, x)=\mathbf{P}_{0}(x), \quad \text { and } \quad \partial_{t} \mathbf{P}(0, x)=\mathbf{P}_{1}(x) \text { on } G
\end{gathered}
$$

are imposed. The electromagnetic field is governed by the classical Maxwell equations, whereas the polarizable medium occupying the set $G$ is modelled as a gas of quantum mechanical systems with two energy levels as described in [5] and [11]. Here $\Omega \subset \mathbb{R}^{3}$ is an arbitrary spatial domain, $G \subset \Omega$ a certain subset of $\Omega$, and $\Gamma_{1} \subset \partial \Omega, \Gamma_{2} \stackrel{\text { def }}{=} \partial \Omega \backslash \Gamma_{1}$. Also, the whole space case $\Omega=\mathbb{R}^{3}$ without boundary condition 1.4 is under consideration.

The unknown functions are the electric and magnetic fields $\mathbf{E}, \mathbf{H}$, which depend on the time $t \geq 0$ and the space variable $x \in \Omega$ and the dielectic polarization $\mathbf{P}$ defined on the set $\mathbb{R}^{+} \times G$. Furthermore, $N$ denotes the difference of the densities of the electrons in the excited and in the ground state. It is also an unknown function defined on $\mathbb{R}^{+} \times G$. In (1.1) the function $\widetilde{\mathbf{P}}$ is the extension of $\mathbf{P}$ on $\mathbb{R} \times \Omega$ defined by zero on the set $\mathbb{R}^{+} \times(\Omega \backslash G)$. The physical meaning of the boundary condition 1.4 is that $\Gamma_{1}$ is perfectly conducting, such that the tangential component of the electric field must vanish.

Received August 1, 2000.

2000 Mathematics Subject Classification. Primary 35Q60, 78A25, 35F25, 35L45. 
The coefficients $\alpha \in L^{\infty}(G), \beta \in L^{\infty}(G), \lambda \in L^{\infty}(G)$, and $\gamma \in L^{\infty}(G)$, which depend on the space variables, take into account the possibly variable dipole moment and the density of the medium as well as the inverse of the relaxation times for the polarization and for the density $N$. The equilibrium density $N_{e} \in L^{\infty}(G)$ is assumed to be positive.

A prescribed external current, $\mathbf{j} \in L^{1}\left((0, \infty), L^{2}(\Omega)\right)$, is included. The nonnegative function $\sigma \in L^{\infty}(\Omega)$ represents the electric conductivity.

In [5], where $G=\Omega=\mathbb{R}^{3}$ and the coefficients do not depend on $x$, it is shown that (1.1)-(1.6) admit a unique strong solution in $C\left(\left[(0, \infty), H^{s}\left(\mathbb{R}^{3}\right)\right)\right.$ for $s \geq 2$. Note that in our case, system (1.1) does not admit classical solutions on all of $(0, \infty) \times \Omega$ due to the discontinuity of $\widetilde{\mathbf{P}}$ on $\Sigma \stackrel{\text { def }}{=}(\partial G) \cap \Omega$, the interface between the polarizable medium and the vacuum region $\Omega \backslash G$. For smooth solutions, (1.1) includes a transmission condition, which requires the continuity of the tangential components of $\mathbf{E}$ and $\mathbf{H}$ on $\Sigma$. In Sec. 3, suitable weak formulation admitting discontinuous solutions to (1.1), (1.2), (1.3) and an existence proof for weak solutions with the properties

$$
\begin{gathered}
(\mathbf{E}, \mathbf{H}) \in C\left([0, \infty), L^{2}\left(\Omega, \mathbb{R}^{6}\right)\right), \\
\mathbf{P} \in W_{\mathrm{loc}}^{2,2}\left([0, \infty), L^{2}\left(G, \mathbb{R}^{3}\right)\right) \cap W_{\mathrm{loc}}^{1,2}\left([0, \infty), L^{\infty}\left(G, \mathbb{R}^{3}\right)\right),
\end{gathered}
$$

and

$$
N(\cdot)-N_{e} \in W_{\mathrm{loc}}^{1,2}\left([0, \infty), L^{2}(G)\right) \cap L_{\mathrm{loc}}^{\infty}\left([0, \infty), L^{\infty}(G)\right)
$$

will be given.

Section 4 is devoted to the investigation of the long-time asymptotic behaviour of the solutions $(\mathbf{E}, \mathbf{H}, \mathbf{P}, N)$ of $(1.1)-(1.6)$. First it is shown that

$$
\lim _{t \rightarrow \infty}\left\|N(t)-N_{e}\right\|_{L^{q}(G)}=0 \quad \text { for all } q \in[1,2) .
$$

Let $\mathcal{N}$ denote the set of all $(\mathbf{f}, \mathbf{g}) \in X \stackrel{\text { def }}{=} L^{2}\left(\Omega, \mathbb{R}^{6}\right)$ that satisfy

$$
\begin{array}{cc}
\operatorname{curl} \mathbf{f}=\operatorname{curl} \mathbf{g}=0 \quad \text { on } \Omega, \\
\vec{n} \wedge \mathbf{f}=0 \quad \text { on } \Gamma_{1}, \\
\vec{n} \wedge \mathbf{g}=0 \quad \text { on } \Gamma_{2},
\end{array}
$$

and

$$
\mathbf{f}=0 \text { on the set } G_{\sigma} .
$$

Here $\Omega \backslash G_{\sigma}$ is the set of vanishing conductivity, i.e., the set of all $x \in \Omega$ with $\sigma(x)=0$.

The main result concerning the asymptotic behavior is that

$$
(\mathbf{E}(t), \mathbf{H}(t)) \stackrel{t \rightarrow \infty}{\longrightarrow}\left(\mathbf{E}_{\infty}, \mathbf{H}_{\infty}\right) \quad \text { in } X \text { weakly }
$$

and

$$
\mathbf{P}(t) \stackrel{t \rightarrow \infty}{\longrightarrow} \mathbf{P}_{\infty} \text { in } L^{2}(G) \text { weakly, }
$$

where the functions $\left(\mathbf{E}_{\infty}, \mathbf{H}_{\infty}\right) \in \mathcal{N}$ and $\mathbf{P} \in L^{2}(G)$ satisfy

$$
\int_{\Omega}\left(\left[\mathbf{E}_{\infty}+\widetilde{\mathbf{P}}_{\infty}\right] \mathbf{f}+\mathbf{H}_{\infty} \mathbf{g}\right) d x=\int_{\Omega}\left(\left[\mathbf{D}_{1} \mathbf{f}+\mathbf{H}_{0} \mathbf{g}\right) d x\right.
$$


for all $(\mathbf{f}, \mathbf{g}) \in \mathcal{N}$ and

$$
\alpha \mathbf{P}_{\infty}=N_{e} \mathbf{E}_{\infty} \quad \text { on } G .
$$

Here $\mathbf{D}_{1} \stackrel{\text { def }}{=} \mathbf{E}_{0}+\widetilde{\mathbf{P}}_{0}-\int_{0}^{\infty} \mathbf{j}(s) d s$ is determined by the initial data and the external current, where $\widetilde{\mathbf{P}}_{0}$ denotes the extension of $\mathbf{P}_{0}$ by zero on $\Omega \backslash G$. By (1.9) the functions $\left(\mathbf{E}_{\infty}, \mathbf{H}_{\infty}\right) \in \mathcal{N}$ and $\mathbf{P}_{\infty} \in L^{2}(G)$ satisfy

$$
\begin{gathered}
\operatorname{curl} \mathbf{E}_{\infty}=\operatorname{curl} \mathbf{H}_{\infty}=0 \quad \text { on } \Omega, \\
\vec{n} \wedge \mathbf{E}_{\infty}=0 \quad \text { on } \Gamma_{1}, \\
\vec{n} \wedge \mathbf{H}_{\infty}=0 \quad \text { on } \Gamma_{2}, \\
\operatorname{div}\left(\mathbf{E}_{\infty}+\widetilde{\mathbf{P}}_{\infty}\right)=\operatorname{div} \mathbf{D}_{1} \quad \text { on } \Omega \backslash G_{\sigma}, \\
\operatorname{div} \mathbf{H}_{\infty}=\operatorname{div} \mathbf{H}_{0} \quad \text { on } \Omega,
\end{gathered}
$$

the boundary conditions

$$
\vec{n}\left(\mathbf{E}_{\infty}+\widetilde{\mathbf{P}}_{\infty}\right)=\vec{n} \mathbf{D}_{1} \quad \text { on } \Gamma_{2} \backslash G_{\sigma}
$$

and

$$
\vec{n} \mathbf{H}_{\infty}=\vec{n} \mathbf{H}_{0} \quad \text { on } \Gamma_{1} .
$$

(This follows from the fact that $(\nabla \varphi, \nabla \psi) \in \mathcal{N}$ for all $\varphi \in C_{0}^{\infty}\left(\mathbb{R}^{3} \backslash\left(\overline{\Gamma_{1}} \cup \overline{G_{\sigma}}\right)\right)$ and $\psi \in C_{0}^{\infty}\left(\mathbb{R}^{3} \backslash \overline{\Gamma_{2}}\right)$.) In the case $\mathbf{j}=0$, the function $\left(\mathbf{E}_{\infty}, \mathbf{H}_{\infty}, \mathbf{P}_{\infty}, N_{e}\right)$ is a stationary state for the system (1.1)-(1.4).

Note that $\mathbf{E}_{\infty}=\mathbf{H}_{\infty}=0$ if the initial data satisfy the condition

$$
\int_{\Omega}\left(\mathbf{D}_{1} \mathbf{f}+\mathbf{H}_{0} \mathbf{g}\right) d x=0 \quad \text { for all }(\mathbf{f}, \mathbf{g}) \in \mathcal{N} .
$$

As a consequence, one obtains decay of the electromagnetic field in the weak $L^{2}(\Omega)$ topology if and only if condition (1.11) is fulfilled. This condition includes

$$
\operatorname{div} \mathbf{D}_{1}=0 \quad \text { on } \Omega \backslash G_{\sigma}
$$

and

$$
\operatorname{div} \mathbf{H}_{0}=0 \quad \text { on } \Omega .
$$

By (1.1), the function $\mathbf{D} \stackrel{\text { def }}{=} \mathbf{E}+\widetilde{\mathbf{P}}$ and $\mathbf{H}$ obey $\operatorname{div} \mathbf{H}(t)=\operatorname{div} \mathbf{H}_{0}=0$ and

$$
\operatorname{div} \mathbf{D}(t)=\operatorname{div}\left[\mathbf{E}_{0}+\widetilde{\mathbf{P}}_{0}-\int_{0}^{t} \mathbf{j}(s) d s\right] \stackrel{t \rightarrow \infty}{\longrightarrow} \operatorname{div} \mathbf{D}_{1}=0 \quad \text { in } \mathcal{D}^{\prime}\left(\Omega \backslash G_{\sigma}\right) .
$$

Hence the physical meaning of condition (1.11) is that the space charge $\rho \stackrel{\text { def }}{=} \operatorname{div} \mathbf{D}$ determined by the initial state $\left(\mathbf{E}_{0}, \mathbf{H}_{0}\right)$ and the prescribed current $\mathbf{j}$ vanishes as $t \rightarrow \infty$ in the nonconducting region.

The basic step of the proof of (1.7) and (1.8) is that the $\omega$-limit set $\omega_{0} \subset L^{2}\left(\Omega, \mathbb{R}^{6}\right)$ of $(\mathbf{E}, \mathbf{H})$ with respect to the weak $L^{2}(\Omega)$ topology satisfies

$$
\omega_{0} \in \mathcal{N}
$$


The proof of (1.12) is based on a result in [8], where weak decay of solutions of certain semilinear hyperbolic systems including Maxwell's equations is shown by identifying the weak $\omega$-limit set of the trajectories; see also [4], [6].

In Sec. 5 , the case where the set $G$ is bounded and $\Omega=\mathbb{R}^{3}$ is considered. It is shown that $\mathbf{u}(t) \stackrel{\text { def }}{=}(\mathbf{E}(t), \mathbf{H}(t))-\left(\mathbf{E}_{\infty}, \mathbf{H}_{\infty}\right)$ obeys

$$
\int_{\{|x| \leq a t\}}|\mathbf{u}|^{2} d x \stackrel{t \rightarrow \infty}{\longrightarrow} 0 \quad \text { for all } \alpha<1
$$

in particular, that the convergence in (1.7) is locally strong in the energy space. The physical meaning of $(1.13)$ is that the wave-packet $\mathbf{u}(t)$ is concentrated near the sphere $|x|=t$ for large times. Furthermore, also strong convergence in (1.8) is shown, i.e.,

$$
\lim _{t \rightarrow \infty}\left\|\mathbf{P}(t)-\mathbf{P}_{\infty}\right\|_{L^{q}(G)}=0 \quad \text { for all } q \in[1,2) .
$$

2. Basic assumptions, definitions. For an arbitrary open set $K \subset \mathbb{R}^{3}$, the space of all infinitely differentiable functions with compact support contained in $K$ is denoted by $C_{0}^{\infty}(K) . H_{\text {curl }}(K)$ is defined as the space of all $\mathbf{E} \in L^{2}\left(K, \mathbb{C}^{3}\right)$ with $\operatorname{curl} \mathbf{E} \in L^{2}(K)$.

Let $\Omega \subset \mathbb{R}^{3}$ be an arbitrary domain and $G \subset \Omega$ an open nonempty set.

Next, let $\sigma \in L^{\infty}(\Omega)$ be a nonnegative function and $\alpha \in L^{\infty}(G), \beta \in L^{\infty}(G), \gamma \in$ $L^{\infty}(G)$, and $N_{e} \in L^{\infty}(G)$ be uniformly positive functions on $G$. Furthermore, let

$$
G_{\sigma} \stackrel{\text { def }}{=}\{x \in \Omega: \sigma(x)>0\} .
$$

In the sequel, we denote by $\underline{\mathbf{w}}_{1} \in \mathbb{C}^{3}$ the first three and by $\underline{\mathbf{w}}_{2} \in \mathbb{C}^{3}$ the last three components of a vector $\mathbf{w} \in \mathbb{C}^{6}$.

Next, some function spaces related to Maxwell's equations with mixed boundary conditions are introduced.

$W_{H}$ denotes the closure of $C_{0}^{\infty}\left(\mathbb{R}^{3} \backslash \overline{\Gamma_{2}}, \mathbb{C}^{3}\right)$ in $H_{\text {curl }}(\Omega)$, where $H_{\text {curl }}(\Omega)$ is the space of all $\mathbf{E} \in L^{2}\left(\Omega, \mathbb{C}^{3}\right)$ with curl $\mathbf{E} \in L^{2}(\Omega) . W_{E}$ denotes the set of all $\mathbf{E} \in H_{\text {curl }}(\Omega)$ such that

$$
\int_{\Omega} \mathbf{E} \operatorname{curl} \mathbf{F}-\mathbf{F} \operatorname{curl} \mathbf{E} d x=0 \quad \text { for all } \mathbf{F} \in W_{H},
$$

which includes a weak formulation of the boundary condition $\vec{n} \wedge \mathbf{E}=0$ on $\Gamma_{1}$; see [7].

Now, the following operators are defined. Let $D(B) \stackrel{\text { def }}{=} W_{E} \times W_{H}$ and let

$$
B(\mathbf{E}, \mathbf{H}) \stackrel{\text { def }}{=}(\operatorname{curl} \mathbf{H},-\operatorname{curl} \mathbf{E}) \quad \text { for }(\mathbf{E}, \mathbf{H}) \in D(B) .
$$

It turns out that $B$ is a densely defined skew selfadjoint operator in the Hilbert space $X \stackrel{\text { def }}{=} L^{2}\left(\Omega, \mathbb{C}^{6}\right)$ endowed with the usual scalar product.

Next, let $\mathcal{N}$ be the set of all $\mathbf{w}=(\mathbf{E}, \mathbf{h}) \in \operatorname{ker} B$ with $\mathbf{E}(x)=0$ for all $x \in G_{\sigma}$.

For $\mathbf{f} \in L_{\text {loc }}^{1}([0, \infty), X)$, a function $\mathbf{u} \in C([0, \infty), X)$ is called a weak solution to the initial boundary value problem

$$
\partial_{t} \underline{\mathbf{u}}_{1}=\operatorname{curl} \underline{\mathbf{u}}_{2}+\underline{\mathbf{f}}_{1}, \quad \partial_{t} \underline{\mathbf{u}}_{2}=-\operatorname{curl} \underline{\mathbf{u}}_{1}+\underline{\mathbf{f}}_{2},
$$

supplemented by the initial boundary conditions

$$
\vec{n} \wedge \underline{\mathbf{u}}_{1}=0 \quad \text { on }(0, \infty) \times \Gamma_{1},
$$


and

$$
\vec{n} \wedge \underline{\mathbf{u}}_{2}=0 \quad \text { on }(0, \infty) \times \Gamma_{2}
$$

if

$$
\frac{d}{d t}\langle\mathbf{u}(t), \mathbf{a}\rangle_{X}=-\langle\mathbf{u}(t), B \mathbf{a}\rangle_{X}+\langle\mathbf{f}(t), \mathbf{a}\rangle_{X} \quad \text { for all } \mathbf{a} \in D(B) .
$$

This means that (2.14) is fulfilled in the sense of distributions, whereas the boundary conditions (2.15) are satisfied in the sense that $\int_{0}^{t} \mathbf{u}(s) d s \in D(B)=W_{E} \times W_{H}$ for all $t \geq 0$. It is well known that (2.16) is equivalent to the variation of constant formula

$$
\mathbf{u}(t)=\exp (t B) \mathbf{u}(0)+\int_{0}^{t} \exp ((t-s) B) \mathbf{f}(s) d s
$$

where $(\exp (t B))_{t \in \mathbb{R}}$ is the unitary group generated by $B$; see [1], [7] and [12]. Equation (2.17) yields the energy estimate

$$
\frac{1}{2} \frac{d}{d t}\|\mathbf{u}(t)\|_{X}^{2}=\langle\mathbf{f}(t), \mathbf{u}(t)\rangle_{X}
$$

Let

$$
\begin{gathered}
\mathbf{j} \in L^{1}\left((0, \infty), L^{2}\left(\Omega, \mathbb{R}^{3}\right)\right) \cap W_{\mathrm{loc}}^{1,1}\left([0, \infty), L^{2}(\Omega)\right), \\
\left(\mathbf{E}_{0}, \mathbf{H}_{0}\right) \in D(B), \quad \mathbf{P}_{0}, \mathbf{P}_{1} \in L^{\infty}(G),
\end{gathered}
$$

and

$$
N_{0} \in L^{\infty}(G)
$$

Furthermore, it is assumed that $\mathbf{P}_{0} \in L^{2}(G), \mathbf{P}_{1} \in L^{2}(G)$, and $N_{0}-N_{e} \in L^{2}(G)$.

3. Existence and uniqueness of weak solutions. First some a priori bounds on the solution $\mathbf{P}, N$ of Eqs. (1.2), (1.3), and (1.6) are given. Suppose that $\mathbf{E} \in$ $C\left([0, \infty), L^{2}\left(\Omega, \mathbb{R}^{3}\right)\right)$ and let $\mathbf{P}, N$ be the solution to $(1.2),(1.3)$, and (1.6). Then

$$
\begin{aligned}
\frac{1}{2} \partial_{t}\left[\gamma(x)\left|\partial_{t} \mathbf{P}(t, x)\right|^{2}+\gamma(x) \alpha(x)|\mathbf{P}(t, x)|^{2}+N(t, x)^{2}\right] \\
=\gamma(x) \partial_{t} \mathbf{P}(t, x)\left[\partial_{t}^{2} \mathbf{P}(t, x)+\alpha(x) \mathbf{P}(t, x)\right] \\
\quad-N(t, x)\left[\lambda(x)\left(N(t, x)-N_{e}(x)\right)+\gamma(x) \partial_{t} \mathbf{P}(t, x) \mathbf{E}(t, x)\right] \\
=-\gamma(x) \beta(x)\left|\partial_{t} \mathbf{P}(t, x)\right|^{2}-\lambda(x) N(t, x)\left(N(t, x)-N_{e}(x)\right) \leq \lambda(x) N_{e}(x)^{2} / 2 .
\end{aligned}
$$

This implies the pointwise bound

$$
|\mathbf{P}(t, x)|+\left|\partial_{t} \mathbf{P}(t, x)\right|+N(t, x) \leq C_{1}\left(1+t^{1 / 2}\right)
$$

with some constant $C_{1} \in(0, \infty)$ independent of $\mathbf{E}$ and $t \geq 0$. In particular, the ordinary initial value problem (1.2), (1.3), and (1.6) admits a global solution $\mathbf{P}, N$ defined on $(0, \infty) \times G$. With $(3.20)$ and $\mathbf{E} \in C\left([0, \infty), L^{2}\left(\Omega, \mathbb{R}^{3}\right)\right)$ one has

$$
N \mathbf{E} \in L_{\mathrm{loc}}^{\infty}\left([0, \infty), L^{2}\left(G, \mathbb{R}^{3}\right)\right) \quad \text { and } \quad \mathbf{E} \partial_{t} \mathbf{P} \in L_{\mathrm{loc}}^{\infty}\left([0, \infty), L^{2}(G)\right) .
$$

Since $\mathbf{P}_{0}, \mathbf{P}_{1} \in L^{2}\left(G, \mathbb{R}^{3}\right),(1.2)$ and (3.21) yield

$$
\mathbf{P} \in W_{\mathrm{loc}}^{2,2}\left([0, \infty), L^{2}\left(G, \mathbb{R}^{3}\right)\right) \cap W_{\mathrm{loc}}^{1,2}\left([0, \infty), L^{\infty}(G)\right) .
$$


Furthermore,

$$
N(\cdot)-N_{e} \in W_{\mathrm{loc}}^{1,2}\left([0, \infty), L^{2}(G)\right) \cap L_{\mathrm{loc}}^{\infty}\left([0, \infty), L^{\infty}(G)\right),
$$

by (1.3) and (3.21), since $N_{0}-N_{e} \in L^{2}\left(G, \mathbb{R}^{3}\right)$.

Now suppose $\mathbf{E} \in C\left([0, \infty), L^{2}\left(\Omega, \mathbb{R}^{3}\right)\right)$ and $\mathbf{F} \in C\left([0, \infty), L^{2}\left(\Omega, \mathbb{R}^{3}\right)\right)$ and let $\mathbf{P}, N$ and $\mathbf{Q}, M$ be the corresponding solutions to (1.2), (1.3), and (1.6). Then

$$
\begin{aligned}
\frac{1}{2} \frac{d}{d t}[ & \left.\left\|\partial_{t} \mathbf{P}(t)-\partial_{t} \mathbf{Q}(t)\right\|_{L^{2}(G)}^{2}+\left\|\gamma^{-1 / 2}(N(t)-M(t))\right\|_{L^{2}(G)}^{2}\right] \\
= & \int_{G}\left(\partial_{t} \mathbf{P}-\partial_{t} \mathbf{Q}\right)\left[-\beta\left(\partial_{t} \mathbf{P}-\partial_{t} \mathbf{Q}\right)-\alpha \mathbf{P}+\alpha \mathbf{Q}+N \mathbf{E}-M \mathbf{F}\right] d x \\
& -\int_{G}(N-M)\left[\gamma^{-1} \lambda(N-M)+\mathbf{E} \partial_{t} \mathbf{P}-\mathbf{F} \partial_{t} \mathbf{Q}\right] d x \\
\leq & C_{2}\left(\left\|\partial_{t} \mathbf{P}(t)-\partial_{t} \mathbf{Q}(t)\right\|_{L^{2}(G)}^{2}+\|\mathbf{P}(t)-\mathbf{Q}(t)\|_{L^{2}(G)}^{2}\right) \\
& +\int_{G}\left(M \partial_{t} \mathbf{P}-N \partial_{t} \mathbf{Q}\right)(\mathbf{E}-\mathbf{F}) d x \\
\leq & C_{2}\left(\left\|\partial_{t} \mathbf{P}(t)-\partial_{t} \mathbf{Q}(t)\right\|_{L^{2}(G)}^{2}+\|\mathbf{P}(t)-\mathbf{Q}(t)\|_{L^{2}(G)}^{2}\right) \\
& +C_{3}\left(1+t^{1 / 2}\right)\left(\left\|\partial_{t} \mathbf{P}(t)-\partial_{t} \mathbf{Q}(t)\right\|_{L^{2}(G)}+\|N(t)-M(t)\|_{L^{2}(G)}\right)\|\mathbf{E}(t)-\mathbf{F}(t)\|_{L^{2}(\Omega)}
\end{aligned}
$$

by (3.20). By Gronwall's lemma, one finds for each time $T>0$ a constant $C_{1, T} \in(0, \infty)$, such that

$$
\left\|\partial_{t} \mathbf{P}-\partial_{t} \mathbf{Q}\right\|_{L^{\infty}\left((0, T), L^{2}(G)\right)} \leq C_{1, T}\|\mathbf{E}-\mathbf{F}\|_{L^{\infty}\left((0, T), L^{2}(\Omega)\right)} .
$$

Let $\mathcal{A}: C([0, \infty), X) \rightarrow C([0, \infty), X)$ be defined by

$$
\begin{aligned}
& (\mathcal{A}(\mathbf{E}, \mathbf{H}))(t)=\exp (t B)\left(\mathbf{E}_{0}, \mathbf{H}_{0}\right) \\
& \quad-\int_{0}^{t} \exp ((t-s) B)\left[\mathcal{R} \partial_{t} \mathbf{P}(s)+(\sigma \mathbf{E}(s)+\mathbf{j}(s), 0)\right] d s,
\end{aligned}
$$

where $\mathbf{P}, N$ solve (1.2), (1.3), and (1.6). Here $\mathcal{R}: L^{2}(G) \rightarrow X$ is defined by

$$
(\mathcal{R} \mathbf{p})(x) \stackrel{\text { def }}{=}(\mathbf{p}(x), 0) \quad \text { if } x \in G
$$

and

$$
(\mathcal{R} \mathbf{p})(x) \stackrel{\text { def }}{=} 0 \quad \text { if } x \in \Omega \backslash G .
$$

Now suppose $(\mathbf{E}, \mathbf{H}) \in C([0, \infty), X)$ and let $\mathbf{P} \in W_{\text {loc }}^{2,2}\left([0, \infty), L^{2}\left(G, \mathbb{R}^{3}\right)\right)$ and $N$ with $N(\cdot)-N_{e} \in W_{\text {loc }}^{1,2}\left([0, \infty), L^{2}(G)\right) \cap L_{\text {loc }}^{\infty}\left([0, \infty), L^{\infty}(G)\right)$ be the solution to $(1.2),(1.3)$, and (1.6). Then (E, $\mathbf{H}, \mathbf{P}, N)$ solves (1.1)-(1.6) (in the sense of 2.16), if

$$
\begin{aligned}
& (\mathbf{E}(t), \mathbf{H}(t))=\exp (t B)\left(\mathbf{E}_{0}, \mathbf{H}_{0}\right) \\
& \quad-\int_{0}^{t} \exp ((t-s) B)\left[\mathcal{R} \partial_{t} \mathbf{P}(s)+(\sigma \mathbf{E}(s)+\mathbf{j}(s), 0)\right] d s
\end{aligned}
$$

i.e.,

$$
\mathcal{A}(\mathbf{E}, \mathbf{H})=(\mathbf{E}, \mathbf{H}) .
$$


Now it follows from the estimates (2.18) and (3.22), using the contraction mapping principle in the space $C([0, T], X)$ with arbitrary large $T>0$, that the fixed point problem 3.24 has a unique solution on each finite time interval $(0, T)$ and hence a unique global solution on $(0, \infty)$.

Theorem 1. Problem (1.1)-(1.6) has a unique weak solution $(\mathbf{E}, \mathbf{H}, \mathbf{P}, N)$ with the properties $(\mathbf{E}, \mathbf{H}) \in C([0, \infty), X), \mathbf{P} \in W_{\mathrm{loc}}^{2,2}\left([0, \infty), L^{2}\left(G, \mathbb{R}^{3}\right)\right) \cap W_{\mathrm{loc}}^{1,2}\left([0, \infty), L^{\infty}(G)\right)$ and $N(\cdot)-N_{e} \in W_{\text {loc }}^{1,2}\left([0, \infty), L^{2}(G)\right) \cap L_{\text {loc }}^{\infty}\left([0, \infty), L^{\infty}(G)\right)$.

By (3.20) one obtains the following $L^{\infty}$ estimate.

LEMma 1. There exists a constant $K_{b}$ independent of $t$, such that

$$
\|\mathbf{P}(t)\|_{L^{\infty}(G)}+\left\|\partial_{t} \mathbf{P}(t)\right\|_{L^{\infty}(G)}+\|N(t)\|_{L^{\infty}(G)} \leq K_{b}\left(1+t^{1 / 2}\right) \text { for all } t \geq 0 .
$$

Next, further estimates on the solution are given using the energy functional

$$
\begin{aligned}
\mathcal{E}(t) \stackrel{\text { def }}{=} & (\| \mathbf{E}(t), \mathbf{H}(t))\left\|_{X}^{2}+\right\| N_{e}^{-1 / 2} \partial_{t} \mathbf{P}(t) \|_{L^{2}(G)}^{2} \\
& \left.+\left\|N_{e}^{-1 / 2} \alpha^{1 / 2} \mathbf{P}(t)\right\|_{L^{2}(G)}^{2}+\left\|\gamma^{-1 / 2} N_{e}^{-1 / 2}\left(N(t)-N_{e}\right)\right\|_{L^{2}(G)}^{2}\right) .
\end{aligned}
$$

The physical meaning of $\mathcal{E}(t)$ is the total energy of the system, including the potential and kinetic energy and the energy of the electromagnetic field.

LEMMA 2. It follows that

$$
\begin{gathered}
(\mathbf{E}, \mathbf{H}) \in L^{\infty}((0, \infty), X), \quad \mathbf{P} \in L^{\infty}\left((0, \infty), L^{2}(G)\right), \\
\partial_{t} \mathbf{P} \in L^{\infty}\left((0, \infty), L^{2}(G)\right) \cap L^{2}\left((0, \infty), L^{2}(G)\right),
\end{gathered}
$$

and

$$
N-N_{e} \in L^{\infty}\left((0, \infty), L^{2}(G)\right) \cap L^{2}\left((0, \infty), L^{2}(G)\right)
$$

Moreover,

$$
\int_{0}^{\infty} \int_{\Omega} \sigma|\mathbf{E}|^{2} d x d t=\int_{0}^{\infty}\left\|\sigma^{1 / 2} \mathbf{E}(t)\right\|_{L^{2}(\Omega)}^{2} d t<\infty .
$$

Proof. First (3.23) and the energy estimate (2.18) yield

$$
\begin{aligned}
\frac{1}{2} \frac{d}{d t} \mathcal{E}(t)=- & \left\langle\mathcal{R} \partial_{t} \mathbf{P}(t)+(\sigma \mathbf{E}(t)+\mathbf{j}(t), 0),(\mathbf{E}(t), \mathbf{H}(t))\right\rangle_{X} \\
& +\int_{G} N_{e}^{-1} \partial_{t} \mathbf{P}\left(\partial_{t}^{2} \mathbf{P}+\alpha \mathbf{P}\right) d x-\int_{G}\left(N_{e}^{-1} N-1\right)\left(\gamma^{-1} \lambda\left(N-N_{e}\right)+\partial_{t} \mathbf{P E}\right) d x \\
=- & \int_{G} N_{e}^{-1} \beta\left|\partial_{t} \mathbf{P}\right|^{2} d x-\int_{G} \lambda \gamma^{-1} N_{e}^{-1}\left|N-N_{e}\right|^{2} d x \\
& \quad-\int_{\Omega} \sigma|\mathbf{E}|^{2} d x-\int_{\Omega} \mathbf{E j} d x \\
\leq & \|(\mathbf{E}(t), \mathbf{H}(t))\|_{X}\|\mathbf{j}(t)\|_{L^{2}(\Omega)}-\left\|\sigma^{1 / 2} \mathbf{E}(t)\right\|_{L^{2}(\Omega)}^{2} \\
& \quad-c_{0}\left\|\partial_{t} \mathbf{P}\right\|_{L^{2}(G)}^{2}-c_{0}\left\|N(t)-N_{e}\right\|_{L^{2}(G)}^{2} .
\end{aligned}
$$

Since $\|\mathbf{j}(\cdot)\|_{L^{2}(\Omega)} \in L^{1}(0, \infty)$, the assertion follows from Gronwall's lemma. 
By the regularity assumption $\mathbf{j} \in W_{\text {loc }}^{1,1}\left([0, \infty), L^{2}(\Omega)\right)$ in $(2.19)$, it follows that $\mathcal{R} \partial_{t} \mathbf{P}(\cdot)$ $+(\mathbf{j}, 0) \in W_{\text {loc }}^{1,1}([0, \infty), X)$. Since also $\left(\mathbf{E}_{0}, \mathbf{H}_{0}\right) \in D(B)$, it follows from the result in [12, Corollary 2.5 , Sec. 4.2] that

$$
(\mathbf{E}, \mathbf{H}) \in C^{1}([0, \infty), X) \cap C([0, \infty), D(B))
$$

is a strong solution of $\partial_{t}(\mathbf{E}(t), \mathbf{H}(t))=B(\mathbf{E}(t), \mathbf{H}(t))-\mathcal{R} \partial_{t} \mathbf{P}(t)-(\sigma \mathbf{E}(t)+\mathbf{j}(t), 0)$. However, it is not clear whether $\left\|\partial_{t}(\mathbf{E}(t), \mathbf{H}(t))\right\|_{X}$ or $\|B(\mathbf{E}(t), \mathbf{H}(t))\|_{X}$ remain bounded for $t \rightarrow \infty$.

4. Weak convergence to stationary states. The next lemma concerns the convergence of the density $N$ to the equilibrium $N_{e}$ as $t \rightarrow \infty$.

Lemma 3. Suppose that, in addition, $N_{0}-N_{e} \in L^{1}(G)$. For all $q \in[1,2)$ one has

$$
\left\|N(t)-N_{e}\right\|_{L^{q}(G)} \stackrel{t \rightarrow \infty}{\rightarrow} 0 .
$$

Proof. The idea is to multiply Eq. (1.3) by the $\operatorname{sign}\left(N-N_{e}\right)$ to obtain an $L^{1}$-estimate:

$$
\begin{aligned}
\frac{d}{d t}\left\|N(t)-N_{e}\right\|_{L^{1}(G)} & =-\int_{G} \operatorname{sign}\left(N-N_{e}\right)\left[\lambda\left(N-N_{e}\right)+\gamma \mathbf{E} \partial_{t} \mathbf{P}\right] \\
& \leq-\left\|\lambda\left(N(t)-N_{e}\right)\right\|_{L^{1}(G)}+\|\left(\mathbf{E}(t), \mathbf{H}(t)\left\|_{X}\right\| \gamma \partial_{t} \mathbf{P}(t) \|_{L^{2}(G)}\right. \\
& \leq-\left\|\lambda\left(N(t)-N_{e}\right)\right\|_{L^{1}(G)}+C_{1}\left\|\partial_{t} \mathbf{P}(t)\right\|_{L^{2}(G)}
\end{aligned}
$$

by Lemma 2 . Hence

$$
\begin{aligned}
\left\|N(t)-N_{e}\right\|_{L^{1}(G)} \leq \| & N_{0}-N_{e} \|_{L^{1}(G)} \exp \left(-t \lambda_{0}\right) \\
& +C_{1} \int_{0}^{t} \exp \left((s-t) \lambda_{0}\right)\left\|\partial_{t} \mathbf{P}(s)\right\|_{L^{2}(G)} d s
\end{aligned}
$$

with $\lambda_{0} \stackrel{\text { def }}{=} \operatorname{ess} \inf \lambda_{0}$. Since $\left\|\partial_{t} \mathbf{P}(\cdot)\right\|_{L^{2}(G)} \in L^{2}(0, \infty)$ by Lemma 2 , this yields

$$
\left\|N(t)-N_{e}\right\|_{L^{1}(G)} \stackrel{t \rightarrow \infty}{\longrightarrow} 0 .
$$

By Lemma 2 again, $\left\|N_{e}-N(t)\right\|_{L^{2}(G)}$ is bounded on $(0, \infty)$. Finally, the assertion follows from (4.30) by interpolation.

The following "unique continuation" principle has been shown in [8], which holds even for arbitrary spatial domains. As in [8] it will be used in the investigation of the weak $\omega$-limit set of the solution of (1.1)--(1.6).

Theorem 2. Suppose that $\mathrm{g} \in X$ obeys

$$
\underline{(\exp (t B) \mathbf{g})}_{1}=0 \text { on } G \text { for all } t \in \mathbb{R} .
$$

Then $\mathbf{g} \in \operatorname{ker} B$.

The above theorem is a suitable modification for not necessarily bounded domains of the unique-continuation principle for the scalar wave equation, which is used in [4], [6], and [13].

Theorem 2 says that each solution $(\mathbf{e}, \mathbf{f}) \in C\left(\mathbb{R}, L^{2}\left(\Omega, \mathbb{R}^{M+N}\right)\right)$ of the evolution equation $\partial_{t}(\mathbf{e}, \mathbf{f})=B(\mathbf{e}, \mathbf{f})$ with the property that $\mathbf{e}(t, x)=0$ for all $t \in \mathbb{R}$ and $x \in G$ satisfies 
$(\mathbf{e}(0), \mathbf{f}(0)) \in \operatorname{ker} B$. In contrast to the unique continuation principle for bounded domains, it is necessary to require the condition $\mathbf{e}(t, x)=0$ on $G$ for all $t \in \mathbb{R}$ and not only for positive times. The basic idea of the proof of Theorem 2 is to show that for each $f \in C_{0}^{\infty}(\mathbb{R} \backslash\{0\})$, the function $f(i B) \mathbf{g}$ is real analytic and vanishes on $G$. This implies $f(i B) \mathbf{g}=0$ for all $f \in C_{0}^{\infty}(\mathbb{R} \backslash\{0\})$ and hence $\mathbf{g} \in \operatorname{ker} B$. (Here the operator $f(i B)$ can be defined by the spectral theorem, since $i B$ is selfadjoint in $L^{2}\left(\Omega, \mathbb{C}^{6}\right)$.)

Let $\omega_{0}$ be the $\omega$-limit set of the trajectory $(\mathbf{E}, \mathbf{H})$ with respect to the weak topology of $X$, i.e., the set of all $\mathbf{G} \in X$, such that there exists a sequence $t_{n} \stackrel{n \rightarrow \infty}{\longrightarrow} \infty$ with $\left(\mathbf{E}\left(t_{n}\right), \mathbf{H}\left(t_{n}\right)\right) \stackrel{n \rightarrow \infty}{\longrightarrow} \mathbf{G}$ in $X$ weakly.

Theorem 3. It follows that $\omega_{0} \subset \mathcal{N}$.

Proof. Suppose $\mathbf{g} \in X$ and $t_{n} \stackrel{n \rightarrow \infty}{\longrightarrow} \infty$ with

$$
\left(\mathbf{E}\left(t_{n}\right), \mathbf{H}\left(t_{n}\right)\right) \stackrel{n \rightarrow \infty}{\longrightarrow} \mathbf{g}
$$

in $X$ weakly. Let $\mathbf{u}_{n}(t) \stackrel{\text { def }}{=}\left(\mathbf{E}\left(t_{n}+t\right), \mathbf{H}\left(t_{n}+t\right)\right) \in X, N_{n}(t) \stackrel{\text { def }}{=} N\left(t_{n}+t\right)$ and $\mathbf{f}_{n}(t) \stackrel{\text { def }}{=}$ $\alpha \mathbf{P}\left(t_{n}+t\right)$ for $n \in \mathbb{N}$. One has, by (3.23),

$$
\begin{aligned}
\mathbf{u}_{n}(t)= & \exp (t B) \mathbf{u}_{n}(0) \\
& -\int_{t_{n}}^{t_{n}+t} \exp \left(\left(t_{n}+t-s\right) B\right)\left[\mathcal{R} \partial_{t} \mathbf{P}(s)+(\sigma \mathbf{E}(s)+\mathbf{j}(s), 0)\right] d s,
\end{aligned}
$$

from which one obtains by Lemma 2 that

$$
\begin{aligned}
\left\|\mathbf{u}_{n}(t)-\exp (t B) \mathbf{u}_{n}(0)\right\|_{X} \leq & \int_{t_{n}}^{t_{n}+t}\left(\left\|\mathcal{R} \partial_{s} \mathbf{P}(s)\right\|_{X}\right. \\
& \left.\quad+\|\sigma \mathbf{E}(s)\|_{L^{2}(\Omega)}+\|\mathbf{j}(s)\|_{L^{2}(\Omega)}\right) d s \stackrel{n \rightarrow \infty}{\longrightarrow} 0
\end{aligned}
$$

for all $t \in \mathbb{R}$ and hence, by (4.32), with $\mathbf{u}_{n}(0)=\left(\mathbf{E}\left(t_{n}\right), \mathbf{H}\left(t_{n}\right)\right)$,

$$
\mathbf{u}_{n}(t) \stackrel{n \rightarrow \infty}{\longrightarrow} \mathbf{u}_{\infty}(t) \stackrel{\text { def }}{=} \exp (t B) \mathbf{g} \text { in } X \text { weakly for all } t \in \mathbb{R} .
$$

Suppose $T>0$. Then Lemma 2 yields

$$
\left\|\mathbf{f}_{n}(t)-\mathbf{f}_{n}(0)\right\|_{L^{2}(G)} \leq C_{1} \int_{\left[t_{n}, t_{n}+t\right]}\left\|\partial_{t} \mathbf{P}(s)\right\|_{L^{2}(G)} d s \stackrel{n \rightarrow \infty}{\longrightarrow} 0
$$

for all $t \in \mathbb{R}$. This implies for all $\varphi \in C_{0}^{\infty}\left((-T, T), L^{2}(G) \cap L^{\infty}(G)\right)$ that

$$
\lim _{n \rightarrow \infty} \int_{-T}^{T} \int_{G} \mathbf{f}_{n}(t) \partial_{t} \varphi(t) d x d t=\lim _{n \rightarrow \infty} \int_{-T}^{T} \int_{G} \mathbf{f}_{n}(0) \partial_{t} \varphi(t) d x d t=0 .
$$

Using $\partial_{t} \mathbf{P} \in L^{2}\left((0, \infty), L^{2}(G)\right)$, again one obtains from (1.2) and (4.35) that

$$
\begin{aligned}
\lim _{n \rightarrow \infty} & \int_{-T}^{T} \int_{G} N_{n}(t) \underline{\mathbf{u}_{n}(t)} \partial_{1} \varphi(t) d x d t \\
& =\lim _{n \rightarrow \infty} \int_{-T}^{T} \int_{G} N\left(t_{n}+t\right) \mathbf{E}\left(t_{n}+t\right) \partial_{t} \varphi(t) d x d t \\
& =\lim _{n \rightarrow \infty} \int_{-T}^{T} \int_{G}\left(\partial_{t}^{2} \mathbf{P}\left(t_{n}+t\right)+\beta \partial_{t} \mathbf{P}\left(t_{n}+t\right)+\mathbf{f}_{n}(t)\right) \partial_{t} \varphi(t) d x d t=0
\end{aligned}
$$


Next

$$
\begin{aligned}
& \left\|N_{e} \underline{\left(\mathbf{u}_{n}\right)} 1-N_{n} \underline{\left(\mathbf{u}_{n}\right)}{ }_{1}\right\|_{L^{2}\left((-T, T), L^{1}(G)\right)} \\
& \quad \leq\left\|\mathbf{u}_{n}\right\|_{L^{\infty}((-T, T), X)}\left(\int_{t_{n}-T}^{t_{n}+T}\left\|N_{e}-N_{n}(t)\right\|_{L^{2}(G)}^{2} d t\right)^{1 / 2} \stackrel{n \rightarrow \infty}{\longrightarrow} 0,
\end{aligned}
$$

since $\left\|N_{e}-N_{n}(\cdot)\right\|_{L^{2}(G)}^{2} \in L^{1}(0, \infty)$ by Lemma 2. Now, one obtains from (4.33), (4.36), and (4.37) that

$$
\begin{aligned}
\int_{-T}^{T} \int_{G} N_{e} \underline{\left(u_{\infty}\right)_{1}}(t) \partial_{t} \varphi(t) d x d t & \left.=\lim _{n \rightarrow \infty} \int_{-T}^{T} \int_{G} N_{e} \underline{\left(\mathbf{u}_{n}\right)(t)}\right)_{1} \partial_{t} \varphi(t) d x d t \\
& =\lim _{n \rightarrow \infty} \int_{-T}^{T} \int_{G} N_{n} \underline{\left(\mathbf{u}_{n}\right)(t)_{1}} \partial_{t} \varphi(t) d x d t=0 .
\end{aligned}
$$

Since $T>0$ and $\varphi \in C_{0}^{\infty}\left((-T, T), L^{2}(G) \cap L^{\infty}(G)\right)$ are chosen arbitrarily, this yields

$$
\partial_{t} \underline{\left(\mathbf{u}_{\infty}\right)_{1}}=0 \text { on } \mathbb{R} \times G .
$$

Because there is no uniform bound for $\left\|B\left(\mathbf{E}\left(t_{n}\right), \mathbf{H}\left(t_{n}\right)\right)\right\|_{X}$ as $n \rightarrow \infty$, one cannot expect that $\mathbf{g} \in D(B)$ at this stage. For this purpose, let $\chi \in C_{0}^{\infty}(\mathbb{R})$ and $\hat{\chi}$ be its Fourier transform. Then

$$
\begin{aligned}
\exp (t B) \chi(i B) \mathbf{g} & =(2 \pi)^{-1 / 2} \int_{\mathbb{R}} \hat{\chi}(\xi) \exp ((t-\xi) B) \mathbf{g} d \xi \\
& =(2 \pi)^{-1 / 2} \int_{\mathbb{R}} \hat{\chi}(\xi) \mathbf{u}_{\infty}(t-\xi) d \xi .
\end{aligned}
$$

Here the operator $f(i B)$ is defined by the spectral theorem, since $i B$ is selfadjoint in $L^{2}\left(\Omega, \mathbb{C}^{6}\right)$. Since $\chi(i B) \mathbf{g} \in D\left(B^{2}\right)$, one obtains from (4.39),

$$
\underline{(\exp (t B) B \chi(i B) \mathbf{g})_{1}}=\partial_{t} \underline{(\exp (t B) \chi(i B) \mathbf{g})_{1}}=0 \quad \text { on } \mathbb{R} \times G .
$$

Invoking Theorem 2, one obtains $B \chi(i B) \mathbf{g} \in \operatorname{ker} B$, and hence $\|B \chi(i B) \mathbf{g}\|_{X}^{2}=$ $-\left\langle\chi(i B) \mathbf{g}, B^{2} \chi(i B) \mathbf{g}\right\rangle_{X}=0$, whence $\chi(i B) \mathbf{g} \in \operatorname{ker} B$. Since $\chi \in C_{0}^{\infty}(\mathbb{R})$ is chosen arbitrarily, it follows that

$$
\mathrm{g} \in \operatorname{ker} B \text {. }
$$

It remains to show that $\underline{\mathrm{g}}_{1}(x)=0$ for all $x \in G_{\sigma}$. By (4.33) and (4.41) one has

$$
\mathbf{u}_{n}(t) \stackrel{n \rightarrow \infty}{\longrightarrow} \mathbf{g} \text { in } X \text { weakly for all } t \in \mathbb{R},
$$

and hence by the dominated convergence theorem,

$$
\int_{0}^{1}\left\langle\mathbf{u}_{n}(t), \mathbf{h}\right\rangle_{X} d t \stackrel{n \rightarrow \infty}{\longrightarrow} \int_{0}^{1}\langle\mathbf{g}, \mathbf{h}\rangle_{X} d t=\langle\mathbf{g}, \mathbf{h}\rangle_{X}
$$

for all $\mathbf{h} \in X$, i.e., $\int_{0}^{1} \mathbf{u}_{n}(t) d t \stackrel{n \rightarrow \infty}{\longrightarrow} \mathbf{g}$ weakly. In particular,

$$
\int_{0}^{1} \sigma^{1 / 2}{\underline{\left(\mathbf{u}_{n}(t)\right)}}_{1} d t \stackrel{n \rightarrow \infty}{\longrightarrow} \sigma^{1 / 2} \underline{\mathbf{g}}_{1} \text { in } L^{2}\left(G_{\sigma}\right) \text { weakly. }
$$


On the other hand, it follows from Lemma 2 that

$$
\left\|\int_{0}^{1} \sigma^{1 / 2}{\underline{\left(\mathbf{u}_{n}(t)\right)_{1}}}_{1} d t\right\|_{L^{2}(\Omega)} \leq\left(\int_{t_{n}}^{1+t_{n}}\left\|\sigma^{1 / 2} \mathbf{E}(t)\right\|_{L^{2}(\Omega)}^{2} d t\right)^{1 / 2} \stackrel{n \rightarrow \infty}{\longrightarrow} 0 .
$$

Now (4.42) and (4.43) yield

$$
\underline{\mathrm{g}}_{1}(x)=0 \text { for all } x \in G_{\sigma} .
$$

By 4.41 the assertion follows.

Let $\mathbf{a}=(\mathbf{f}, \mathbf{g}) \in \mathcal{N}$, i.e, $\mathbf{a} \in \operatorname{ker} B$ with $\mathbf{f}(x)=0$ for all $x \in G_{\sigma}$. By the definition of the set $G_{\sigma}$ one has $\langle(\sigma \mathbf{E}(t), 0), \mathbf{a})_{X}=\int_{\Omega} \sigma \mathbf{E f} d x=0$ for all $t \geq 0$. Now it follows from (3.23) that

$$
\begin{aligned}
\langle(\mathbf{E}(t), \mathbf{H}(t)), \mathbf{a}\rangle_{X}= & \left\langle\exp (t B)\left(\mathbf{E}_{0}, \mathbf{H}_{0}\right)\right. \\
& \left.\quad-\int_{0}^{t} \exp ((t-s) B)\left[(\sigma \mathbf{E}(s)+\mathbf{j}(s), 0)+\partial_{t} \mathcal{R} \mathbf{P}(s)\right] d s, \mathbf{a}\right\rangle_{X} \\
= & \left\langle\left(\mathbf{E}_{0}, \mathbf{H}_{0}\right)-\int_{0}^{t}\left[(\sigma \mathbf{E}(s)+\mathbf{j}(s), 0)+\partial_{t} \mathcal{R} \mathbf{P}(s)\right] d s, \mathbf{a}\right\rangle_{X}
\end{aligned}
$$

and hence

$$
\langle(\mathbf{E}(t), \mathbf{H}(t))+\mathcal{R} \mathbf{P}(t), \mathbf{a}\rangle_{X}=\left\langle\mathbf{a}_{0}+\mathbf{J}(t), \mathbf{a}\right\rangle_{X}
$$

for all $\mathbf{a} \in \mathcal{N}$, with

$$
\mathbf{a}_{0} \stackrel{\text { def }}{=}\left(\mathbf{E}_{0}, \mathbf{H}_{0}\right)+\mathcal{R} \mathbf{P}_{0}-\int_{0}^{\infty}(\mathbf{j}(s), 0) d s
$$

and

$$
\mathbf{J}(t) \stackrel{\text { def }}{=} \int_{t}^{\infty}(\mathbf{j}(s), 0) d s .
$$

Now, the main theorem concerning the weak convergence to stationary states can be proved.

ThEOREM 4. There exist uniquely determined functions $\left(\mathbf{E}_{\infty}, \mathbf{H}_{\infty}\right) \in \mathcal{N}$ and $\mathbf{P} \in L^{2}(G)$ with

$$
\left\langle\left(\mathbf{E}_{\infty}, \mathbf{H}_{\infty}\right)+\mathcal{R} \mathbf{P}_{\infty}, \mathbf{a}\right\rangle_{X}=\left\langle\mathbf{a}_{0}, \mathbf{a}\right\rangle_{X} \quad \text { for all } \mathbf{a} \in \mathcal{N}
$$

and

$$
\alpha \mathbf{P}_{\infty}=N_{e} \mathbf{E}_{\infty} \quad \text { on } G .
$$

Moreover,

$$
(\mathbf{E}(t), \mathbf{H}(t)) \stackrel{t \rightarrow \infty}{\longrightarrow}\left(\mathbf{E}_{\infty}, \mathbf{H}_{\infty}\right) \text { in } X \text { weakly }
$$

and

$$
\mathbf{P}(t) \stackrel{t \rightarrow \infty}{\longrightarrow} \mathbf{P}_{\infty} \text { in } L^{2}(G) \text { weakly. }
$$


Proof. Suppose $t_{n} \stackrel{n \rightarrow \infty}{\longrightarrow} \infty$ with

$$
\left(\mathbf{E}\left(t_{n}\right), \mathbf{H}\left(t_{n}\right)\right) \stackrel{n \rightarrow \infty}{\longrightarrow}\left(\mathbf{E}_{\infty}, \mathbf{H}_{\infty}\right)
$$

in $X$ weakly and

$$
\mathbf{P}\left(t_{n}\right) \stackrel{n \rightarrow \infty}{\longrightarrow} \mathbf{P}_{\infty} \text { in } L^{2}(G) \text { weakly. }
$$

First, Theorem 3 yields

$$
\left(\mathbf{E}_{\infty}, \mathbf{H}_{\infty}\right) \in \mathcal{N} \subset \operatorname{ker} B .
$$

Furthermore, (4.45) follows from (4.44), (4.49), and (4.50). Next it is shown that $\left(\mathbf{E}_{\infty}, \mathbf{H}_{\infty}\right) \in \operatorname{ker} B$ and $\mathbf{P} \in L^{2}(G)$ satisfy $(4.46)$.

Let $\mathbf{u}_{n}(t)$ and $N_{n}$ for $n \in \mathbb{N}$ be as in the proof of Theorem 3. Invoking (4.33) and (4.51), one obtains

$$
\mathbf{u}_{n}(t) \stackrel{n \rightarrow \infty}{\longrightarrow} \mathbf{u}_{\infty}(t) \stackrel{\text { def }}{=} \exp (t B)\left(\mathbf{E}_{\infty}, \mathbf{H}_{\infty}\right)=\left(\mathbf{E}_{\infty}, \mathbf{H}_{\infty}\right)
$$

in $X$ weakly for all $t \in \mathbb{R}$.

Since $\partial_{t} \mathbf{P} \in L^{2}\left((0, \infty), L^{2}(G)\right)$, by Lemma 2 one has

$$
\left\|\mathbf{P}\left(t_{n}+t\right)-\mathbf{P}\left(t_{n}\right)\right\|_{L^{2}(G)} \leq \int_{\left[t_{n}, t_{n}+t\right]}\left\|\partial_{t} \mathbf{P}(s)\right\|_{L^{2}(G)} d s \stackrel{n \rightarrow \infty}{\longrightarrow} 0
$$

for all $t \in \mathbb{R}$. By (4.50) this implies

$$
\mathbf{P}\left(t_{n}+t\right) \stackrel{n \rightarrow \infty}{\longrightarrow} \mathbf{P}_{\infty} \text { in } L^{2}(G) \text { weakly for all } t \in \mathbb{R} .
$$

Suppose $\varphi \in C_{0}^{\infty}\left((-1,1), L^{2}(G) \cap L^{\infty}(G)\right)$. Then one obtains from (4.52) and (4.54) as in (4.38) that

$$
\begin{aligned}
\int_{-1}^{1} \int_{G} N_{e} \mathbf{E}_{\infty} \varphi(t) d x d t & =\lim _{n \rightarrow \infty} \int_{-1}^{1} \int_{G} N_{e} \underline{\left(\mathbf{u}_{n}\right)(t)}{ }_{1} \varphi(t) d x d t \\
& =\lim _{n \rightarrow \infty} \int_{-1}^{1} \int_{G} N_{n} \underline{\left(\mathbf{u}_{n}\right)(t)}{ }_{1} \varphi(t) d x d t \\
& =\lim _{n \rightarrow \infty} \int_{-1}^{1} \int_{G}\left(\partial_{t}^{2} \mathbf{P}\left(t_{n}+t\right)+\beta \partial_{t} \mathbf{P}\left(t_{n}+t\right)+\alpha \mathbf{P}_{n}(t)\right) \varphi(t) d x d t \\
& =\int_{-1}^{1} \int_{G} \alpha \mathbf{P}_{\infty} \varphi(t) d x d t
\end{aligned}
$$

In the last step, $\partial_{t} \mathbf{P} \in L^{2}\left((0, \infty), L^{2}(G)\right)$ is used again. Since $\varphi \in C_{0}^{\infty}\left((-1,1), L^{2}(G) \cap\right.$ $\left.L^{\infty}(G)\right)$ is arbitrary, this yields (4.46).

In order to show that $\left(\mathbf{E}_{\infty}, \mathbf{H}_{\infty}\right) \in \operatorname{ker} B$ and $\mathbf{P} \in L^{2}(G)$ are uniquely determined, suppose that $\left(\mathbf{F}_{\infty}, \mathbf{G}_{\infty}\right) \in \operatorname{ker} B$ and $\mathbf{Q} \in L^{2}(G)$ also obey (4.45) and (4.46). Then

$$
\begin{aligned}
\left\|\left(\mathbf{E}_{\infty}-\mathbf{F}_{\infty}, \mathbf{H}_{\infty}-\mathbf{G}_{\infty}\right)\right\|_{X}^{2} & =-\left\langle\mathcal{R}\left(\mathbf{P}_{\infty}-\mathbf{Q}_{\infty}\right),\left(\mathbf{E}_{\infty}-\mathbf{F}_{\infty}, \mathbf{H}_{\infty}-\mathbf{G}_{\infty}\right)\right\rangle_{X} \\
& =-\int_{G}\left(\mathbf{E}_{\infty}-\mathbf{F}_{\infty}\right)\left(\mathbf{P}_{\infty}-\mathbf{Q}_{\infty}\right) d x \\
& =-\int_{G} N_{e}^{-1} \alpha\left[\mathbf{P}_{\infty}-\mathbf{Q}_{\infty}\right]\left[\mathbf{P}_{\infty}-\mathbf{Q}_{\infty}\right] d x \leq 0 .
\end{aligned}
$$


This completes the proof of uniqueness. In particular, it follows that $\left(\mathbf{E}_{\infty}, \mathbf{H}_{\infty}, \mathbf{P}_{\infty}\right)$ is the only possible accumulation point of $(\mathbf{E}, \mathbf{H}, \mathbf{P})$, whence (4.47) and (4.48).

5. Local strong convergence in the case $\Omega=\mathbb{R}^{3}$. Throughout this section it is assumed that the set $G$ is bounded and $\Omega=\mathbb{R}^{3}$. Furthermore, it is assumed that the external current $\mathbf{j}$ and $\sigma$ are located in a fixed finite ball, i.e.,

$$
G_{\sigma} \subset B_{R_{1}} \text { and } \mathbf{j}(t, x)=0 \text { for all } t \in(0, \infty), \quad x \in \mathbb{R}^{3} \backslash B_{R_{1}},
$$

with some $R_{1}>0$.

In this case, $X=L^{2}\left(\mathbb{R}^{3}\right)$ and $D(B)=H_{\text {curl }}\left(\mathbb{R}^{3}\right) \times H_{\text {curl }}\left(\mathbb{R}^{3}\right)$. Let $Q$ be the orthogonal projector on $(\operatorname{ker} B)^{\perp}=\overline{\operatorname{ran} B}$, which consists of all $\mathbf{u} \in L^{2}\left(\mathbb{R}^{3}\right)$ with $\operatorname{div} \underline{\mathbf{u}}_{j}=0$. Then $1-Q$ is the orthogonal projector on $\operatorname{ker} B$ consisting of all $\mathbf{u} \in L^{2}\left(\mathbb{R}^{3}\right)$ with curl $\underline{\mathbf{u}}_{j}=0$.

The main goal of this section is to prove the decay property (1.13). Since $B(\mathbf{E}(t), \mathbf{H}(t))$ is generally not bounded in $X$ as $t \rightarrow \infty$, it is a priori not clear whether $\{(\dot{\mathbf{E}}(t), \mathbf{H}(t)): t \geq$ $0\}$ or at least the divergence free part $\{Q(\mathbf{E}(t), \mathbf{H}(t)): t \geq 0\}$ are locally precompact.

For all $\mathbf{a} \in \operatorname{ker} B$ one has

$$
\begin{aligned}
\langle(\mathbf{E}(t), \mathbf{H}(t)), \mathbf{a}\rangle_{X}= & \left\langle\exp (t B)\left(\mathbf{E}_{0}, \mathbf{H}_{0}\right)\right. \\
& \left.\quad-\int_{0}^{t} \exp ((t-s) B)\left[\mathcal{R} \partial_{t} \mathbf{P}(s)+(\sigma \mathbf{E}(s)+\mathbf{j}(s), 0)\right] d s, \mathbf{a}\right\rangle_{X} \\
= & \left\langle\left(\mathbf{E}_{0}, \mathbf{H}_{0}\right)+\mathcal{R} \mathbf{P}_{0}-\mathcal{R} \mathbf{P}(t)-\int_{0}^{t}[(\sigma \mathbf{E}(s)+\mathbf{j}(s), 0)] d s, \mathbf{a}\right\rangle_{X}
\end{aligned}
$$

and hence

$$
(1-Q)[(\mathbf{E}(t), \mathbf{H}(t))+\mathcal{R} \mathbf{P}(t)]=(1-Q)\left[\mathbf{a}_{0}+\mathbf{J}(t)-\mathbf{G}(t)\right] d s
$$

with

$$
\begin{aligned}
& \mathbf{a}_{0} \stackrel{\text { def }}{=}\left(\mathbf{E}_{0}, \mathbf{H}_{0}\right)+\mathcal{R} \mathbf{P}_{0}-\int_{0}^{\infty}(\mathbf{j}(s), 0) d s, \\
& \mathbf{J}(t) \stackrel{\text { def }}{=} \int_{t}^{\infty}(\mathbf{j}(s), 0) d s
\end{aligned}
$$

and

$$
\mathbf{G}(t) \stackrel{\text { def }}{=} \int_{0}^{t}(\sigma \mathbf{E}(s), 0) d s .
$$

LEMMA 4. It follows that

$$
\begin{gathered}
t^{-1} \int_{0}^{t}\langle(\mathbf{E}(s), \mathbf{H}(s)), Q \mathcal{R} \mathbf{P}(s)\rangle_{X} d s \stackrel{t \rightarrow \infty}{\longrightarrow} 0, \\
t^{-1} \int_{0}^{t}\langle(\mathbf{E}(s), \mathbf{H}(s)), \mathbf{G}(s)\rangle_{X} d s \stackrel{t \rightarrow \infty}{\longrightarrow} 0
\end{gathered}
$$


and

$$
t^{-1} \int_{0}^{t}\langle(\mathbf{E}(s), \mathbf{H}(s)), Q \mathbf{G}(s)\rangle_{X} d s \stackrel{t \rightarrow \infty}{\longrightarrow} 0 .
$$

Proof. Let $\mathbf{F}(t) \stackrel{\text { def }}{=} Q(\mathbf{E}(t), \mathbf{H}(t))$ and $\mathbf{A}(t) \stackrel{\text { def }}{=} \int_{0}^{t} \underline{\mathbf{F}}_{1}(s) d s$. Since $\mathbf{F}(t) \in(\operatorname{ker} B)^{\perp}$ one has

$$
\operatorname{div} \mathbf{A}(t)=0 \quad \text { on } \mathbb{R}^{3}
$$

Let $\mathbf{g} \in H_{\text {curl }}\left(\mathbb{R}^{3}\right)$. Then it follows from (2.16) and (3.23) that

$$
\begin{aligned}
\int_{\mathbb{R}^{3}} \mathbf{A}(t) \operatorname{curl} \mathbf{g} d x & =\langle(\mathbf{A}(t), 0), B(0, \mathbf{g})\rangle_{X} \\
& =\int_{0}^{t}\langle Q(\mathbf{E}(s), \mathbf{H}(s)), B(0, \mathbf{g})\rangle_{X} d s \\
& =\int_{0}^{t}\langle(\mathbf{E}(s), \mathbf{H}(s)), B(0, \mathbf{g})\rangle_{X} d s \\
& =-\int_{0}^{t}\left[\frac{d}{d t}\langle(\mathbf{E}(s), \mathbf{H}(s)),(0, \mathbf{g})\rangle_{X}\right. \\
& =\left\langle\left(\mathbf{E}_{0}, \mathbf{H}_{0}\right),(0, \mathbf{g})\right\rangle_{X}-\langle(\mathbf{E}(t), \mathbf{H}(t)),(0, \mathbf{g})\rangle_{X} \\
& =\int_{\mathbb{R}^{3}}\left(\mathbf{H}_{0}-\mathbf{H}(t)\right) \mathbf{g} d x .
\end{aligned}
$$

Hence

$$
\operatorname{curl} \mathbf{A}(t)=\mathbf{H}_{0}(t)-\mathbf{H}(t) \in L^{2}\left(\mathbb{R}^{3}\right)
$$

which implies by (5.58) and Sobolev's inequality that $\mathbf{A}(t) \in L^{6}\left(\mathbb{R}^{3}\right)$ and

$$
\|\mathbf{A}(t)\|_{L^{6}\left(\mathbb{R}^{3}\right)} \leq K_{3}\|\operatorname{curl} \mathbf{A}(t)\|_{L^{2}\left(\mathbb{R}^{3}\right)}=K_{3}\|\mathbf{H}(0)-\mathbf{H}(t)\|_{L^{2}\left(\mathbb{R}^{3}\right)} .
$$

Now, it follows from Lemma 2 and the previous estimate that

$$
\|\mathbf{A}(t)\|_{L^{6}\left(\mathbb{R}^{3}\right)} \leq C_{1} \quad \text { for all } t \in(0, \infty)
$$

with some constant $C_{1}$ independent of $t$. 
Using Hölder's inequality and the assumption that $G$ is bounded, one obtains from Lemma 2 and (5.59) that

$$
\begin{aligned}
t^{-1} \int_{0}^{t}\langle Q(\mathbf{E}(s), \mathbf{H}(s)), \mathcal{R} \mathbf{P}(s)\rangle_{X} d s \\
\quad=t^{-1} \int_{0}^{t} \int_{G} \underline{\mathbf{F}}_{1}(s) \mathbf{P}(s) d x d s \\
\quad=t^{-1} \int_{0}^{t} \int_{G} \partial_{t} \mathbf{A}(s) \mathbf{P}(s) d x d s \\
\quad=t^{-1} \int_{G} \mathbf{A}(t) \mathbf{P}(t) d x-t^{-1} \int_{0}^{t} \int_{G} \mathbf{A}(s) \partial_{t} \mathbf{P}(s) d x d s \\
\leq t^{-1}\|\mathbf{A}(t)\|_{L^{6}(G)}\|\mathbf{P}(t)\|_{L^{6 / 5}(G)}+t^{-1} \int_{0}^{t}\|\mathbf{A}(s)\|_{L^{6}(G)}\left\|\partial_{t} \mathbf{P}(s)\right\|_{L^{6 / 5}(G)} d s \\
\quad \leq C_{2} t^{-1}\|\mathbf{P}(t)\|_{L^{2}(G)}+C_{2} t^{-1} \int_{0}^{t}\left\|\partial_{t} \mathbf{P}(s)\right\|_{L^{2}(G)} d s \\
\quad \leq C_{2} t^{-1}\left(\left\|\mathbf{P}_{0}\right\|_{L^{2}(G)}+2 \int_{0}^{t}\left\|\partial_{t} \mathbf{P}(s)\right\|_{L^{2}(G)} d s\right) \\
\leq C_{3} t^{-1}+2 C_{2} t^{-1 / 2}\left\|\partial_{s} \mathbf{P}\right\|_{L^{2}\left((0, \infty), L^{2}(G)\right)}^{\stackrel{t \rightarrow \infty}{\longrightarrow} 0,}
\end{aligned}
$$

whence the first assertion. Next,

$$
\begin{aligned}
\langle(\mathbf{E}(t), \mathbf{H}(t)), \mathbf{G}(t)\rangle_{X} & \leq \int_{0}^{t} \int_{\Omega} \sigma|\mathbf{E}(s) \| \mathbf{E}(t)| d x d s \\
& \leq \int_{0}^{t}\left\|\sigma^{1 / 2} \mathbf{E}(s)\right\|_{L^{2}(\Omega)} d s\left\|\sigma^{1 / 2} \mathbf{E}(t)\right\|_{L^{2}(\Omega)} \\
& \leq t^{1 / 2}\left(\int_{0}^{t}\left\|\sigma^{1 / 2} \mathbf{E}(s)\right\|_{L^{2}(\Omega)}^{2} d s\right)^{1 / 2}\left\|\sigma^{1 / 2} \mathbf{E}(t)\right\|_{L^{2}(\Omega)} \\
& \leq C_{4} t^{1 / 2}\left\|\sigma^{1 / 2} \mathbf{E}(t)\right\|_{L^{2}(\Omega)}
\end{aligned}
$$

For all $T>0$ one obtains

$$
\begin{aligned}
& t^{-1} \int_{1}^{t}\langle(\mathbf{E}(s), \mathbf{H}(s)), \mathbf{G}(s)\rangle_{X} d s \\
& \quad \leq t^{-1} \int_{1}^{T}\langle(\mathbf{E}(s), \mathbf{H}(s)), \mathbf{G}(s)\rangle_{X} d s+C_{4} t^{-1} \int_{T}^{t} s^{1 / 2}\left\|\sigma^{1 / 2} \mathbf{E}(s)\right\|_{L^{2}(\Omega)} d s \\
& \quad \leq t^{-1} \int_{1}^{T}\langle(\mathbf{E}(s), \mathbf{H}(s)), \mathbf{G}(s)\rangle_{X} d s+C_{4}\left(\int_{T}^{t}\left\|\sigma^{1 / 2} \mathbf{E}(s)\right\|_{L^{2}(\Omega)}^{2} d s\right)^{1 / 2}
\end{aligned}
$$

and hence by Lemma 2 ,

$$
\limsup _{t \rightarrow \infty}\left(t^{-1} \int_{1}^{t}\langle(\mathbf{E}(s), \mathbf{H}(s)), \mathbf{G}(s)\rangle_{X} d s\right) \leq C_{4}\left(\int_{T}^{\infty}\left\|\sigma^{1 / 2} \mathbf{E}(s)\right\|_{L^{2}(\Omega)}^{2} d s\right)^{1 / 2}
$$

for all $T>0$, which implies the second assertion. 
Next, one obtains from Lemma 2, (5.56), (5.59), and Hölder's inequality with $\mathbf{A}(t) \stackrel{\text { def }}{=}$ $\int_{0}^{t} \underline{\mathbf{F}}_{1}(s) d s$ as above that

$$
\begin{aligned}
t^{-1} \int_{0}^{t} & \langle Q(\mathbf{E}(s), \mathbf{H}(s)), \mathbf{G}(s)\rangle_{X} d s \\
& =t^{-1} \int_{0}^{t} \int_{\mathbb{R}^{3}} \underline{\mathbf{F}}_{1}(s) \mathbf{G}_{1}(s) d x d s=t^{-1} \int_{0}^{t} \int_{\mathbb{R}^{3}} \partial_{s} \mathbf{A}(s) \mathbf{G}_{1}(s) d x d s \\
& =t^{-1}\left[\int_{\mathbb{R}^{3}} \mathbf{A}(t) \mathbf{G}_{1}(t) d x-\int_{0}^{t} \int_{\mathbb{R}^{3}} \mathbf{A}(s) \sigma \mathbf{E}(s) d x d s\right] \\
& \leq t^{-1}\|\mathbf{A}(t)\|_{L^{6}\left(G_{\sigma}\right)}\|\mathbf{G}(t)\|_{L^{6 / 5}\left(\mathbf{G}_{\sigma}\right)}+t^{-1} \int_{0}^{t}\|\mathbf{A}(s)\|_{L^{6}\left(G_{\sigma}\right)}\|\sigma \mathbf{E}(s)\|_{L^{6 / 5}\left(G_{\sigma}\right)} d s \\
& \leq C_{5} t^{-1}\|\mathbf{G}(t)\|_{X}+C_{5} t^{-1} \int_{0}^{t}\left\|\sigma^{1 / 2} \mathbf{E}(s)\right\|_{L^{2}(\Omega)} d s \\
& \leq C_{6} t^{-1} \int_{0}^{t}\left\|\sigma^{1 / 2} \mathbf{E}(s)\right\|_{L^{2}(\Omega)} d s \leq C_{6} t^{-1 / 2}\left\|\sigma^{1 / 2} \mathbf{E}\right\|_{L^{2}\left((0, \infty), L^{2}(\Omega)\right)} \stackrel{t \rightarrow \infty}{\longrightarrow} 0 .
\end{aligned}
$$

This completes the proof.

In what follows, let $\mathbf{u}(s) \stackrel{\text { def }}{=}(\mathbf{E}(s), \mathbf{H}(s))-\left(\mathbf{E}_{\infty}, \mathbf{H}_{\infty}\right)$.

Using Lemma $2,(3.27),\|\mathbf{j}(\cdot)\|_{X} \in L^{1}(0, \infty)$, and (3.28), one obtains $\frac{d}{d t} \mathcal{E}(t) \in L^{1}(0, \infty)$, which implies the existence of the limit

$$
\begin{aligned}
\mathcal{E}_{\infty} \stackrel{\text { def }}{=} \lim _{t \rightarrow \infty} \mathcal{E}(t)=\lim _{t \rightarrow \infty}\left(\|(\mathbf{E}(t), \mathbf{H}(t))\|_{X}^{2}+\left\|N_{e}^{-1 / 2} \partial_{t} \mathbf{P}(t)\right\|_{L^{2}(G)}^{2}\right. \\
\left.\quad+\left\|N_{e}^{-1 / 2} \alpha^{1 / 2} \mathbf{P}(t)\right\|_{L^{2}(G)}^{2}+\left\|\gamma^{-1 / 2} N_{e}^{-1 / 2}\left(N(s)-N_{e}\right)\right\|_{L^{2}(G)}^{2}\right) d s .
\end{aligned}
$$

By Lemma 2 again, it follows that

$$
\mathcal{E}_{\infty}=\lim _{t \rightarrow \infty} t^{-1} \int_{0}^{t}\left(\|(\mathbf{E}(s), \mathbf{H}(s))\|_{X}^{2}+\left\|N_{e}^{-1 / 2} \alpha^{1 / 2} \mathbf{P}(s)\right\|_{L^{2}(G)}^{2}\right) d s .
$$

LEMMA 5. It follows that

$$
\begin{aligned}
\lim _{t \rightarrow \infty} t^{-1} \int_{0}^{t}\|Q \mathbf{u}(s)\|_{X}^{2} d s & =\lim _{t \rightarrow \infty} t^{-1} \int_{0}^{t} \| Q\left(\mathbf{E}(s), \mathbf{H}(s) \|_{X}^{2} d s\right. \\
& =\mathcal{E}_{\infty}-\left\langle\left(\mathbf{E}_{\infty}, \mathbf{H}_{\infty}\right), \mathbf{a}_{0}\right\rangle_{X} \geq \underset{t \rightarrow \infty}{\limsup }\|\mathbf{u}(t)\|_{X}^{2} .
\end{aligned}
$$

Proof. Since $\left(\mathbf{E}_{\infty}, \mathbf{H}_{\infty}\right) \in \mathcal{N} \subset \operatorname{ker} B$, it follows from (5.57) that

$$
\begin{aligned}
\|Q \mathbf{u}(t)\|_{X}^{2} & =\|Q(\mathbf{E}(t), \mathbf{H}(t))\|_{X}^{2}=\|(\mathbf{E}(t), \mathbf{H}(t))\|_{X}^{2}-\|(1-Q)(\mathbf{E}(t), \mathbf{H}(t))\|_{X}^{2} \\
& =\|(\mathbf{E}(t), \mathbf{H}(t))\|_{X}^{2}-\left\langle(\mathbf{E}(t), \mathbf{H}(t)),(1-Q)\left[\mathbf{a}_{0}+\mathbf{J}(t)-\mathbf{G}(t)-\mathcal{R} \mathbf{P}(t)\right]\right\rangle_{X} \\
& =\|(\mathbf{E}(t), \mathbf{H}(t))\|_{X}^{2}+a_{1}(t)-\left\langle(\mathbf{E}(t), \mathbf{H}(t)),(1-Q) \mathbf{a}_{0}\right\rangle_{X}+\int_{G} \mathbf{E}(t) \mathbf{P}(t) d x
\end{aligned}
$$

with

$$
a_{1}(t) \stackrel{\text { def }}{=}-\langle(\mathbf{E}(t), \mathbf{H}(t)),(1-Q) \mathbf{G}(t)+Q \mathcal{R} \mathbf{P}(t)-(1-Q) \mathbf{J}(t)\rangle_{X}
$$


By Lemma 4 one has

$$
\lim _{t \rightarrow \infty} t^{-1} \int_{0}^{t} a_{1}(s) d s=0
$$

Next

$$
\begin{aligned}
\|Q \mathbf{u}(t)\|_{X}^{2}= & a_{1}(t)+a_{2}(t)+\|(\mathbf{E}(t), \mathbf{H}(t))\|_{X}^{2}-\left\langle(\mathbf{E}(t), \mathbf{H}(t)),(1-Q) \mathbf{a}_{0}\right\rangle_{X} \\
& +\int_{G} N_{e}^{-1}\left[\partial_{t}^{2} \mathbf{P}(t)+\beta \partial_{t} \mathbf{P}(t)+\alpha \mathbf{P}(t)\right] \mathbf{P}(t) d x \\
= & a_{1}(t)+a_{2}(t)+a_{3}(t)+\|(\mathbf{E}(t), \mathbf{H}(t))\|_{X}^{2} \\
& +\int_{G} N_{e}^{-1} \alpha|\mathbf{P}(t)|^{2} d x-\left\langle(\mathbf{E}(t), \mathbf{H}(t)),(1-Q) \mathbf{a}_{0}\right\rangle_{X}
\end{aligned}
$$

with

$$
a_{2}(t) \stackrel{\text { def }}{=} \int_{G}\left(1-N(t) / N_{e}\right) \mathbf{E}(t) \mathbf{P}(t) d x
$$

and

$$
a_{3}(t) \stackrel{\text { def }}{=} \int_{G} N_{e}^{-1}\left[\partial_{t}^{2} \mathbf{P}(t)+\beta \partial_{t} \mathbf{P}(t)\right] \mathbf{P}(t) d x
$$

For all $T>0$, one obtains by Lemma 1 and Lemma 2,

$$
\begin{aligned}
\left|t^{-1} \int_{1}^{t} a_{2}(s) d s\right| & \\
\leq & t^{-1} \int_{1}^{T}\left|a_{2}(s)\right| d s \\
& \quad+t^{-1} \int_{T}^{t}\left\|1-N(s) / N_{e}\right\|_{L^{2}(G)}\|\mathbf{E}(s)\|_{L^{2}(G)}\|\mathbf{P}(s)\|_{L^{\infty}(G)} d s \\
\leq & t^{-1} \int_{1}^{T}\left|a_{2}(s)\right| d s+C_{1} t^{-1} \int_{T}^{t} s^{1 / 2}\left\|N(s)-N_{e}\right\|_{L^{2}(G)} d s \\
\leq & t^{-1} \int_{1}^{T}\left|a_{2}(s)\right| d s+C_{1}\left(\int_{T}^{\infty}\left\|N(s)-N_{e}\right\|_{L^{2}(G)}^{2} d s\right)^{1 / 2}
\end{aligned}
$$

and hence

$$
\limsup _{t \rightarrow \infty}\left|t^{-1} \int_{1}^{t} a_{2}(s) d s\right| \leq C_{1}\left(\int_{T}^{\infty}\left\|N(s)-N_{e}\right\|_{L^{2}(G)}^{2} d s\right)^{1 / 2}
$$

for all $T>0$, which implies that

$$
\lim _{t \rightarrow \infty} t^{-1} \int_{0}^{t} a_{2}(s) d s=0
$$


By Lemma 2 again, it follows that

$$
\begin{aligned}
t^{-1} \int_{0}^{t} a_{3}(s) d s=t^{-1}\left[\int_{G}\right. & N_{e}^{-1} \mathbf{P}(t) \partial_{t} \mathbf{P}(t) d x-\int_{G} N_{e}^{-1} \mathbf{P}_{0} \mathbf{P}_{1} d x \\
& -\int_{0}^{t} \int_{G} N_{e}^{-1}\left|\partial_{t} \mathbf{P}(s)\right|^{2} d x d s \\
& \left.+\frac{1}{2} \int_{G} N_{e}^{-1} \beta|\mathbf{P}(t)|^{2} d x-\frac{1}{2} \int_{G} N_{e}^{-1} \beta\left|\mathbf{P}_{0}\right|^{2} d x\right] \stackrel{t \rightarrow \infty}{\longrightarrow} 0 .
\end{aligned}
$$

Now, it follows from (5.63) and (5.64)-(5.67) that

$$
\begin{aligned}
\lim _{t \rightarrow \infty} & t^{-1} \int_{0}^{t}\|Q \mathbf{u}(s)\|_{X}^{2} d s \\
= & \lim _{t \rightarrow \infty} t^{-1} \int_{0}^{t}\left[\|(\mathbf{E}(s), \mathbf{H}(s))\|_{X}^{2}+\int_{G} N_{e}^{-1} \alpha|\mathbf{P}(s)|^{2} d x\right] d s \\
& -\lim _{t \rightarrow \infty} t^{-1} \int_{0}^{t}\left\langle(\mathbf{E}(s), \mathbf{H}(s)),(1-Q) \mathbf{a}_{0}\right\rangle_{X} d s \\
= & \mathcal{E}_{\infty}-\lim _{t \rightarrow \infty} t^{-1} \int_{0}^{t}\left\langle(\mathbf{E}(s), \mathbf{H}(s)),(1-Q) \mathbf{a}_{0}\right\rangle_{X} d s
\end{aligned}
$$

By (4.48) one has

$$
\begin{aligned}
\mathcal{E}_{\infty} & \geq \limsup _{t \rightarrow \infty}\left(\|(\mathbf{E}(t), \mathbf{H}(t))\|_{X}^{2}+\left\|N_{e}^{-1 / 2} \alpha^{1 / 2} \mathbf{P}(t)\right\|_{L^{2}(G)}^{2}\right) \\
& \geq \limsup _{t \rightarrow \infty}\|(\mathbf{E}(t), \mathbf{H}(t))\|_{X}^{2}+\int_{G} N_{e}^{-1} \alpha\left|\mathbf{P}_{\infty}\right|^{2} d x .
\end{aligned}
$$

By taking $\mathbf{a}=\left(\mathbf{E}_{\infty}, \mathbf{H}_{\infty}\right) \in \mathcal{N},(4.45)$ and (4.46) yield

$$
\begin{aligned}
\left\langle\mathbf{a}_{0},\left(\mathbf{E}_{\infty}, \mathbf{H}_{\infty}\right)\right\rangle_{X} & =\left\langle\left(\mathbf{E}_{\infty}, \mathbf{H}_{\infty}\right)+\mathcal{R} \mathbf{P}_{\infty},\left(\mathbf{E}_{\infty}, \mathbf{H}_{\infty}\right)\right\rangle_{X} \\
& =\left\|\left(\mathbf{E}_{\infty}, \mathbf{H}_{\infty}\right)\right\|_{X}^{2}+\int_{G} N_{e}^{-1} \alpha\left|\mathbf{P}_{\infty}\right|^{2} d x .
\end{aligned}
$$

Finally, it follows from (5.68), (5.69), (5.70), and Theorem 4 that

$$
\begin{aligned}
\lim _{t \rightarrow \infty} t^{-1} \int_{0}^{t}\|Q \mathbf{u}(s)\|_{X}^{2} d s & =\mathcal{E}_{\infty}-\left\langle\left(\mathbf{E}_{\infty}, \mathbf{H}_{\infty}\right), \mathbf{a}_{0}\right\rangle_{X} \\
& \geq \limsup _{t \rightarrow \infty}\|(\mathbf{E}(t), \mathbf{H}(t))\|_{X}^{2}-\left\|\left(\mathbf{E}_{\infty}, \mathbf{H}_{\infty}\right)\right\|_{X}^{2} \\
& =\limsup _{t \rightarrow \infty}\|\mathbf{u}(t)\|_{X}^{2} .
\end{aligned}
$$

In the last step, (4.47) is used again.

The following estimate will be used in what follows.

Lemma 6. There exists a constant $K_{1} \in(0, \infty)$ such that

$$
\|(1+|x|) Q \mathbf{f}\|_{L^{2}\left(\mathbb{R}^{3}\right)} \leq K_{1}\|\mathbf{f}\|_{L^{2}\left(\mathbb{R}^{3}\right)}
$$

for all $\mathbf{f} \in C_{0}^{\infty}\left(B_{R_{1}}\right)$. Here $R_{1}$ is as in assumption (5.56). 
Proof. Let $\mathbf{F} \stackrel{\text { def }}{=}(1+|x|) Q \mathbf{f}-Q((1+|x|) \mathbf{f})$. Then

$$
B \mathbf{F}=|x|^{-1} S Q \mathbf{f}+(1+|x|) B \mathbf{f}-B((1+|x|) \mathbf{f})=|x|^{-1} S[Q \mathbf{f}-\mathbf{f}]
$$

and hence

$$
\|B \mathbf{F}\|_{L^{2}\left(\mathbb{R}^{3}\right)} \leq\|(Q-1) \mathbf{f}\|_{L^{2}\left(\mathbb{R}^{3}\right)} \leq\|\mathbf{f}\|_{L^{2}\left(\mathbb{R}^{3}\right)}
$$

with $S \mathbf{w} \stackrel{\text { def }}{=}\left(-x \wedge \underline{\mathbf{w}}_{2}, x \wedge \underline{\mathbf{w}}_{1}\right)$. A similar estimate using $\operatorname{div} \underline{(Q \mathbf{f})}_{j}=0$ yields

$$
\left\|\operatorname{div} \underline{\mathbf{F}}_{j}\right\|_{L^{2}\left(\mathbb{R}^{3}\right)}=\left\|\underline{(Q \mathbf{f})}{ }_{j}\right\|_{L^{2}\left(\mathbb{R}^{3}\right)} \leq\|\mathbf{f}\|_{L^{2}\left(\mathbb{R}^{3}\right)} .
$$

By Sobolev's inequality one obtains

$$
\|\mathbf{F}\|_{L^{6}\left(\mathbb{R}^{3}\right)} \leq C_{2}\left(\left\|\operatorname{curl} \underline{\mathbf{F}}_{j}\right\|_{L^{2}\left(\mathbb{R}^{3}\right)}+\left\|\operatorname{div} \underline{\mathbf{F}}_{j}\right\|_{L^{2}\left(\mathbb{R}^{3}\right)}\right) \leq C_{3}\|\mathbf{f}\|_{L^{2}\left(\mathbb{R}^{3}\right)}
$$

This means that the commutator $[Q,(1+|x|)]$ is a bounded operator from $L^{2}\left(\mathbb{R}^{3}\right)$ to $L^{6}\left(\mathbb{R}^{3}\right)$. By duality it follows that it is also a bounded operator from $L^{6 / 5}\left(\mathbb{R}^{3}\right)$ to $L^{2}\left(\mathbb{R}^{3}\right)$, i.e.,

$$
\|[Q,(1+|x|)] \mathbf{f}\|_{L^{2}\left(\mathbb{R}^{3}\right)} \leq C_{3}\|\mathbf{f}\|_{L^{6 / 5}\left(\mathbb{R}^{3}\right)} .
$$

Therefore, Hölders inequality yields

$$
\begin{aligned}
\|(1+|x|) Q \mathbf{f}\|_{L^{2}\left(\mathbb{R}^{3}\right)} & \leq\|[Q,(1+|x|)] \mathbf{f}\|_{L^{2}\left(\mathbb{R}^{3}\right)}+\|Q((1+|x|) \mathbf{f})\|_{L^{2}\left(\mathbb{R}^{3}\right)} \\
& \leq C_{3}\|\mathbf{f}\|_{L^{6 / 5}\left(\mathbb{R}^{3}\right)}+\|(1+|x|) \mathbf{f}\|_{L^{2}\left(\mathbb{R}^{3}\right)} \\
& \leq K_{1}\|\mathbf{f}\|_{L^{2}\left(\mathbb{R}^{3}\right)}
\end{aligned}
$$

for all $\mathbf{f} \in C_{0}^{\infty}\left(B_{R_{1}}\right)$.

Since $(\mathbf{E}(t), \mathbf{H}(t))$ solves the homogeneous Maxwell equations outside $B_{R_{1}}$, Lemma 7 follows from an energy estimate.

LEMMA 7. For all $b>1$, one has

$$
\int_{\{|x| \geq b t\}}|(\mathbf{E}(t), \mathbf{H}(t))|^{2} d x \stackrel{t \rightarrow \infty}{\longrightarrow} 0 .
$$

Theorem 5. Suppose $g \in C_{0}^{\infty}(\mathbb{R})$ with $g(u)=1$ on a neighbourhood of $[0,1]$. Then

$$
\lim _{t \rightarrow \infty} t^{-1}\langle S g(|x| / t) Q \mathbf{u}(t), Q \mathbf{u}(t)\rangle_{X}=\mathcal{E}_{\infty}-\left\langle\left(\mathbf{E}_{\infty}, \mathbf{H}_{\infty}\right), \mathbf{a}_{0}\right\rangle_{X} \geq \limsup _{t \rightarrow \infty}\|\mathbf{u}(t)\|_{X}^{2}
$$

with $S \mathbf{w} \stackrel{\text { def }}{=}\left(-x \wedge \underline{\mathbf{w}}_{2}, x \wedge \underline{\mathbf{w}}_{1}\right)$.

Proof. Since $\left(\mathbf{E}_{\infty}, \mathbf{H}_{\infty}\right) \in \mathcal{N} \subset \operatorname{ker} B$, it follows by $(3.29)$ that $\mathbf{u} \in C([0, \infty), X)$ is a strong solution of

$$
\partial_{t} \mathbf{u}(t)=B \mathbf{u}(t)-\mathcal{R} \partial_{t} \mathbf{P}(t)-(\sigma \mathbf{E}(s)+\mathbf{j}(s), 0)
$$

Define

$$
F(t) \stackrel{\text { def }}{=}\langle S g(|x| / t) Q \mathbf{u}(t), Q \mathbf{u}(t)\rangle_{X}
$$


Then

$$
\begin{aligned}
F^{\prime}(t)= & 2\left\langle S g(|x| / t) Q \mathbf{u}(t), Q\left(B \mathbf{u}(t)-\mathcal{R} \partial_{t} \mathbf{P}(t)-(\sigma \mathbf{E}(s)+\mathbf{j}(s), 0)\right)\right\rangle_{X} \\
& \quad-t^{-2}\left\langle S|x| g^{\prime}(|x| / t) Q \mathbf{u}(t), Q \mathbf{u}(t)\right\rangle_{X} \\
= & \left.\sum_{j=1}^{2} h_{j}(t)+2\langle S g(|x| / t) Q \mathbf{u}(t)), B Q \mathbf{u}(t)\right\rangle_{X} .
\end{aligned}
$$

Here

$$
\begin{aligned}
& h_{1}(t) \stackrel{\text { def }}{=}-2\left\langle S g(|x| / t) Q \mathbf{u}(t), Q\left(\mathcal{R} \partial_{t} \mathbf{P}(t)+(\sigma \mathbf{E}(t)+\mathbf{j}(t), 0)\right)\right\rangle_{X}, \\
& h_{2}(t) \stackrel{\text { def }}{=}-t^{-2}\left\langle S|x| g^{\prime}(|x| / t) Q \mathbf{u}(t), Q \mathbf{u}(t)\right\rangle_{X} .
\end{aligned}
$$

For $\mathbf{f} \in(\operatorname{ker} B)^{\perp} \cap D(B) \subset H^{1}\left(\mathbb{R}^{3}\right)$ one has $\operatorname{div} \underline{\mathbf{f}}_{j}=0$. Therefore, it follows from the identity $x \wedge \operatorname{curl} \mathbf{a}=\nabla(x \mathbf{a})-\mathbf{a}-(x \nabla) \mathbf{a}$ that

$$
\begin{aligned}
& 2\langle S g(|x| / t) \mathbf{f}, B \mathbf{f}\rangle_{X} \\
&=2 \int_{\mathbb{R}^{3}} g(|x| / t) \mathbf{f} \cdot\left(x \wedge \operatorname{curl} \underline{\mathbf{f}}_{1}, x \wedge \operatorname{curl} \underline{\mathbf{f}}_{2}\right) d x \\
&=2 \int_{\mathbb{R}^{3}} g(|x| / t) \mathbf{f} \cdot\left(\nabla\left[x \underline{\mathbf{f}}_{1}\right], \nabla\left[x \underline{\mathbf{f}}_{2}\right]\right) d x \\
&-2 \int_{\mathbb{R}^{3}} g(|x| / t)|\mathbf{f}|^{2} d x-\int_{\mathbb{R}^{3}} g(|x| / t)(x \nabla)|\mathbf{f}|^{2} d x \\
&=-2 t^{-1}\left\langle\widetilde{S} g^{\prime}(|x| / t) \mathbf{f}, \mathbf{f}\right\rangle_{X}+\left\langle\left[g(|x| / t)+t^{-1}|x| g^{\prime}(|x| / t)\right] \mathbf{f}, \mathbf{f}\right\rangle_{X}
\end{aligned}
$$

with $\widetilde{S} \mathbf{f} \stackrel{\text { def }}{=}|x|^{-1}\left(\left[x \underline{\mathbf{f}}_{1}\right] x,\left[x \underline{\mathbf{f}}_{2}\right] x\right)$. Hence

$$
\begin{aligned}
& 2\langle S g(|x| / t) Q \mathbf{u}(t)), B Q \mathbf{u}(t)\rangle_{X} \\
& \quad=\left\langle\left[g(|x| / t)+t^{-1}|x| g^{\prime}(|x| / t)\right] Q \mathbf{u}(t), Q \mathbf{u}(t)\right\rangle_{X}-2 t^{-1}\left\langle\widetilde{S} g^{\prime}(|x| / t) Q \mathbf{u}(t), Q \mathbf{u}(t)\right\rangle_{X} .
\end{aligned}
$$

With (5.73)-(5.75) it follows that

$$
F^{\prime}(t)=\|Q \mathbf{u}(t)\|_{X}^{2}+h_{1}(t)+h_{3}(t),
$$

where

$$
\begin{aligned}
h_{3}(t) \stackrel{\text { def }}{=}\left\langle\left[g(|x| / t)-1+t^{-1}|x| g^{\prime}(|x| / t)\right] Q \mathbf{u}(t), Q \mathbf{u}(t)\right\rangle_{X} \\
\\
\quad-t^{-1}\left\langle\left(2 \widetilde{S}+t^{-1}|x| S\right) g^{\prime}(|x| / t) Q \mathbf{u}(t), Q \mathbf{u}(t)\right\rangle_{X} .
\end{aligned}
$$

In the following estimates, the $C_{j}$ are constants independent of $s$. Lemma 2 and Lemma 6 yield, by assumption (5.56),

$$
\begin{aligned}
\left|h_{1}(s)\right| & \leq C_{1} \|(1+|x|) Q\left(\mathcal{R} \partial_{t} \mathbf{P}(s)+(\sigma(\mathbf{E}(s)+\mathbf{j}(s), 0)) \|_{L^{2}\left(\mathbb{R}^{3}\right)}\right. \\
& \leq K_{1} C_{1}\left\|\mathcal{R} \partial_{t} \mathbf{P}(s)+(\sigma \mathbf{E}(s)+\mathbf{j}(s), 0)\right\|_{L^{2}\left(B_{R_{1}}\right)} .
\end{aligned}
$$

By Lemma 2 again, one has $\left\|\mathcal{R} \partial_{t} \mathbf{P}(\cdot)+(\sigma \mathbf{E}(\cdot)+\mathbf{j}(s), 0)\right\|_{L^{2}\left(B_{R_{1}}\right)} \in L^{2}(0, \infty)$, which implies

$$
t^{-1} \int_{1}^{t}\left|h_{1}(s)\right| d s \stackrel{t \rightarrow \infty}{\longrightarrow} 0 .
$$


Next, Lemma 5 yields

$$
\begin{aligned}
\lim _{t \rightarrow \infty} t^{-1} \int_{0}^{t}\|(1-Q) \mathbf{u}(s)\|_{X}^{2} d s= & \lim _{t \rightarrow \infty} t^{-1} \int_{0}^{t}\left(\|\mathbf{u}(s)\|_{X}^{2}-\|Q \mathbf{u}(s)\|_{X}^{2}\right) d s \\
& \leq \limsup _{t \rightarrow \infty}\|\mathbf{u}(t)\|_{X}^{2}-\lim _{t \rightarrow \infty} t^{-1} \int_{0}^{t}\|Q \mathbf{u}(s)\|_{X}^{2} d s=0 .
\end{aligned}
$$

Together with Lemma 7 , this implies that, for all $b>1$,

$$
\lim _{t \rightarrow \infty} t^{-1} \int_{0}^{t}\|Q \mathbf{u}(s)\|_{L^{2}(\{|x| \geq b s\})} d s=\lim _{t \rightarrow \infty} t^{-1} \int_{0}^{t}\|\mathbf{u}(s)\|_{L^{2}(\{|x| \geq b s\})} d s=0 .
$$

Since $g^{\prime}(|x| / t)=0$ and $g(|x| / t)=1$ for $|x| \leq b t$ with some $b>1,(5.77)$ and (5.79) yield

$$
t^{-1} \int_{0}^{t}\left|h_{3}(s)\right| d s \leq C_{5} t^{-1} \int_{0}^{t}\|Q \mathbf{u}(s)\|_{L^{2}(\{|x| \geq b s\})} d s \stackrel{t \rightarrow \infty}{\longrightarrow} 0 .
$$

Now, it follows from (5.76)-(5.80) and Lemma 5 that

$$
\begin{aligned}
\lim _{t \rightarrow \infty} t^{-1} F(t) & =\lim _{t \rightarrow \infty} t^{-1} \int_{1}^{t} F^{\prime}(s) d s \\
& =\lim _{t \rightarrow \infty} t^{-1} \int_{1}^{t}\|Q \mathbf{u}(s)\|_{X}^{2} d s \\
& =\mathcal{E}_{\infty}-\left\langle\left(\mathbf{E}_{\infty}, \mathbf{H}_{\infty}\right), \mathbf{a}_{0}\right\rangle_{X} \geq \limsup _{t \rightarrow \infty}\|\mathbf{u}(t)\|_{X}^{2} .
\end{aligned}
$$

This completes the proof.

Now the main result of this section, (1.13), can be proved.

ThEOREM 6. For all $a<1$, one has

$$
\|\mathbf{u}(t)\|_{L^{2}\left(B_{a t}\right)} \stackrel{t \rightarrow \infty}{\longrightarrow} 0
$$

and

$$
\|(1-Q) \mathbf{u}(t)\|_{L^{2}\left(\mathbb{R}^{3}\right)} \stackrel{t \rightarrow \infty}{\longrightarrow} 0 .
$$

Proof. Suppose $\delta>0$. Choose $g \in C_{0}^{\infty}(\mathbb{R},[0, \infty))$ with $g(y)=1$ on $[0,1+\delta / 2]$ and $g(u)=0$ for all $u>1+\delta$. Then

$$
\begin{aligned}
\|(1-Q) \mathbf{u}(t)\|_{X}^{2} & =\|\mathbf{u}(t)\|_{X}^{2}-\|Q \mathbf{u}(t)\|_{X}^{2} \\
& \leq\|\mathbf{u}(t)\|_{X}^{2}-(1+\delta)^{-1} t^{-1}\langle S g(|x| / t) Q \mathbf{u}(t), Q \mathbf{u}(t)\rangle_{X}
\end{aligned}
$$

and hence, by Theorem 5 ,

$$
\limsup _{t \rightarrow \infty}\|(1-Q) \mathbf{u}(t)\|_{X}^{2} \leq\left(1-(1+\delta)^{-1}\right) \limsup _{t \rightarrow \infty}\|\mathbf{u}(t)\|_{X}^{2} .
$$

By letting $\delta \rightarrow 0$, this yields

$$
\lim _{t \rightarrow \infty}\|(1-Q) \mathbf{u}(t)\|_{X}^{2}=0 .
$$

Now it follows from Lemma 7, Theorem 5, and (5.82) that, for all $b>1$,

$$
\begin{aligned}
& \lim _{t \rightarrow \infty} t^{-1}\left\langle S \chi_{\{|x| \leq b t\}} \mathbf{u}(t)\right., \mathbf{u}(t)\rangle_{X}=\lim _{t \rightarrow \infty} t^{-1}\langle S g(|x| / t) \mathbf{u}(t), \mathbf{u}(t)\rangle_{X} \\
&=\lim _{t \rightarrow \infty} t^{-1}\langle S g(|x| / t) Q \mathbf{u}(t), Q \mathbf{u}(t)\rangle_{X} \geq \limsup _{t \rightarrow \infty}\|\mathbf{u}(t)\|_{X}^{2} .
\end{aligned}
$$


Here $g \in C_{0}^{\infty}(\mathbb{R},[0, \infty))$ with $g(y)=1$ on $[0, b]$ and $g(y)=0$ for all $y>2 b$ is chosen.

Let $b>1$. Then one obtains, from (5.83),

$$
\begin{aligned}
\int_{B_{a t}}|\mathbf{u}(t)|^{2} d x & \leq \int_{B_{b t}}|\mathbf{u}(t)|^{2} d x-b^{-1} t^{-1} \int_{\{a t \leq|x| \leq b t\}}|x \| \mathbf{u}(t)|^{2} d x \\
& \leq\|\mathbf{u}(t)\|_{X}^{2}-b^{-1} t^{-1} \int_{B_{b t}}|x| \|\left.\mathbf{u}(t)\right|^{2} d x+b^{-1} a \int_{B_{a t}}|\mathbf{u}(t)|^{2} d x \\
& \leq\|\mathbf{u}(t)\|_{X}^{2}-b^{-1} t^{-1}\left\langle S \chi_{\{|x| \leq b t\}} \mathbf{u}(t), \mathbf{u}(t)\right\rangle_{X}+a \int_{B_{a t}}|\mathbf{u}(t)|^{2} d x
\end{aligned}
$$

Hence

$$
(1-a) \int_{B_{a t}}|\mathbf{u}(t)|^{2} d x \leq\|\mathbf{u}(t)\|_{X}^{2}-b^{-1} t^{-1}\left\langle S \chi_{\{|x| \leq b t\}} \mathbf{u}(t), \mathbf{u}(t)\right\rangle_{X} .
$$

Next, it follows from (5.83) that

$$
(1-a) \varlimsup_{t \rightarrow \infty} \int_{B_{a t}}|\mathbf{u}(t)|^{2} d x \leq\left(1-b^{-1}\right) \limsup _{t \rightarrow \infty}\|\mathbf{u}(t)\|_{X}^{2} \text { for all } b>1 .
$$

By letting $b \rightarrow 1$ this yields

$$
\|\mathbf{u}(t)\|_{L^{2}\left(B_{a t}\right)} \stackrel{t \rightarrow \infty}{\longrightarrow} 0
$$

Finally, the strong convergence of $\mathbf{P}(s)-\mathbf{P}_{\infty}$ is proved.

Lemma 8. For all $q \in[1,2)$ one has

$$
\left\|\mathbf{P}(t)-\mathbf{P}_{\infty}\right\|_{L^{q}(G)} \stackrel{t \rightarrow \infty}{\longrightarrow} 0 .
$$

Proof. By (4.46) and Eq. (1.2), the function $\mathbf{Q}(s) \stackrel{\text { def }}{=} \mathbf{P}(s)-\mathbf{P}_{\infty}$ obeys

$$
\begin{aligned}
\partial_{t}^{2} \mathbf{Q}+\beta \partial_{t} \mathbf{Q}+\alpha \mathbf{Q} & =N \mathbf{E}-N_{e} \mathbf{E}_{\infty} \\
& =\left(N-N_{e}\right) \mathbf{E}-N_{e}\left(\mathbf{E}-\mathbf{E}_{\infty}\right) .
\end{aligned}
$$

Since $\left\|N(\cdot)-N_{e}\right\|_{L^{2}(G)} \in L^{2}(0, \infty)$ and $\|\mathbf{E}(\cdot)\|_{L^{2}(G)} \in L^{\infty}(0, \infty)$ by Lemma 2 , one has

$$
\left\|\left(N(\cdot)-N_{e}\right) \mathbf{E}(\cdot)\right\|_{L^{1}(G)} \leq\left\|N(\cdot)-N_{e}\right\|_{L^{2}(G)}\|\mathbf{E}(\cdot)\|_{L^{2}(G)} \in L^{2}(0, \infty) .
$$

Theorem 6 yields, by the boundedness of the set $G$,

$$
\left\|N_{e}\left(\mathbf{E}(t)-\mathbf{E}_{\infty}\right)\right\|_{L^{1}(G)} \leq C_{1}\left\|\mathbf{E}(t)-\mathbf{E}_{\infty}\right\|_{L^{2}(G)} \stackrel{t \rightarrow \infty}{\longrightarrow} 0 .
$$

As in the proof of Lemma 3, it follows easily from (5.84), (5.85), and (5.86) that

$$
\left\|\mathbf{P}(t)-\mathbf{P}_{\infty}\right\|_{L^{1}(G)} \stackrel{t \rightarrow \infty}{\longrightarrow} 0 .
$$

By Lemma 2 again, $\left\|\mathbf{P}(t)-\mathbf{P}_{\infty}\right\|_{L^{2}(G)}$ is bounded on $(0, \infty)$. Finally, the assertion follows from (5.87) by interpolation.

REMARK 1. Suppose that the initial data obey

$$
\operatorname{div} \mathbf{D}_{1}=\operatorname{div} \mathbf{H}_{0}=0 \quad \text { on } \mathbb{R}^{3} .
$$

By the definition of $B$ and $Q$, this means

$$
\mathbf{a}_{0}=\left(\mathbf{D}_{1}, \mathbf{H}_{0}\right) \in(\operatorname{ker} B)^{\perp} \subset \mathcal{N}^{\perp},
$$


in particular, $(1-Q) \mathbf{a}_{0}=0$ and condition (1.11) is fulfilled. From (5.68) one obtains directly, without using Theorem 4, that

$$
\lim _{t \rightarrow \infty} t^{-1} \int_{0}^{t}\|Q \mathbf{u}(s)\|_{X}^{2} d s=\mathcal{E}_{\infty} \geq \limsup _{t \rightarrow \infty}\|(\mathbf{E}(t), \mathbf{H}(t))\|_{X}^{2}=\limsup _{t \rightarrow \infty}\|\mathbf{u}(t)\|_{X}^{2},
$$

i.e., the assertion of Lemma 5. This means that Theorem 5 and Theorem 6 can be proved under condition (5.88) without using the results of Sec. 4.

\section{REFERENCES}

[1] J. M. Ball, Strongly continuous semigroups, weak solutions and the variation of constants formula, Proc. Amer. Math. Soc. 63, 370-373 (1977)

[2] R. Boyd, Nonlinear Optics, Academic Press, New York, 1992

[3] G. Carbou and P. Fabrie, Time average in micromagnetism, J. Differential Equations 147, 383-409 (1998)

[4] C. M. Dafermos, Asymptotic behavior of solutions of evolution equations, Nonlinear Evolution Equations, Academic Press, New York, 1978, pp. 103-123

[5] P. Donnat and J. Rauch, Global solvability of the Maxwell-Bloch equations from nonlinear optics, Arch. Rational Mech. Anal. 136, 291-303 (1996)

[6] A. Haraux, Stabilization of trajectories for some weakly damped hyperbolic equations, J. Differential Equations 59, 145-154 (1985)

[7] F. Jochmann, Convergence to stationary states of solutions of the transient drift diffusion equations for semiconductor devices with prescribed currents, Asymptotic Anal. 18, 67-109 (1998)

[8] F. Jochmann, Asymptotic behaviour of solutions to a class of semilinear hyperbolic systems in arbitrary domains, J. Differential Equations 60, 439-466 (2000)

[9] J. L. Joly, G. Metivier and J. Rauch, Global solvability of the anharmonic oscillator model from nonlinear optics, SIAM J. Math. Anal. 27, 905-913 (1996)

[10] L. D. Landau and E. M. Lifshitz, Electrodynamics of Continuous Media, Pergamon Press, New York, 1960

[11] R. Pantell and H. Puthoff, Fundamentals of quantum electronics, John Wiley and Sons, New York, 1969

[12] A. Pazy, Semigroups of Linear Operators and Applications to Partial Differential Equations, Springer, New York, 1983

[13] M. Slemrod, Weak asymptotic decay via a relaxed invariance principle for a wave equation with nonlinear nonmonotone damping, Proc. Roy. Soc. Edinburgh 113A, 87-97 (1989) 